\title{
Rayleigh-Taylor Instability within Sediment Layers Due to Gas Retention: Preliminary Theory and Experiments
}

\author{
PA Gauglitz \\ BE Wells \\ SD Rassat \\ WC Buchmiller
}

March 2013

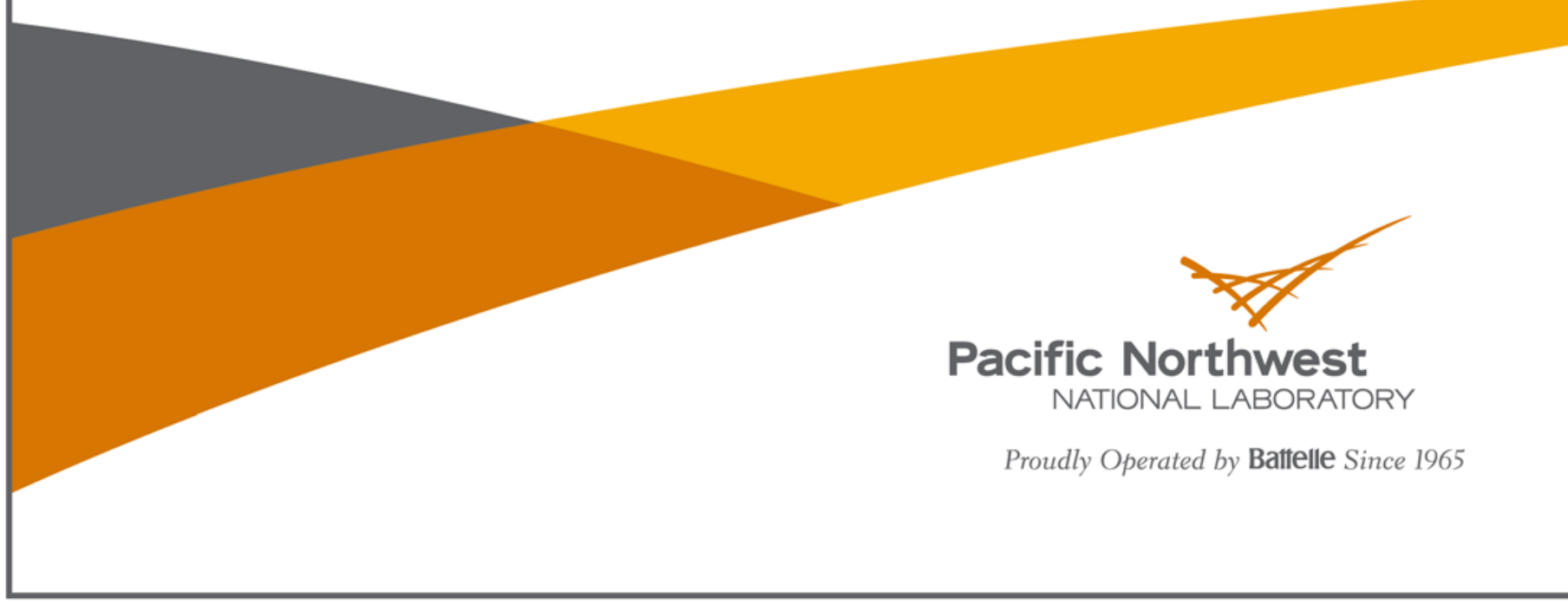




\title{
DISCLAIMER
}

This report was prepared as an account of work sponsored by an agency of the United States Government. Neither the United States Government nor any agency thereof, nor Battelle Memorial Institute, nor any of their employees, makes any warranty, express or implied, or assumes any legal liability or responsibility for the accuracy, completeness, or usefulness of any information, apparatus, product, or process disclosed, or represents that its use would not infringe privately owned rights. Reference herein to any specific commercial product, process, or service by trade name, trademark, manufacturer, or otherwise does not necessarily constitute or imply its endorsement, recommendation, or favoring by the United States Government or any agency thereof, or Battelle Memorial Institute. The views and opinions of authors expressed herein do not necessarily state or reflect those of the United States Government or any agency thereof.

\author{
PACIFIC NORTHWEST NATIONAL LABORATORY \\ operated by \\ BATTELLE \\ for the \\ UNITED STATES DEPARTMENT OF ENERGY \\ under Contract DE-AC05-76RL01830
}

Printed in the United States of America

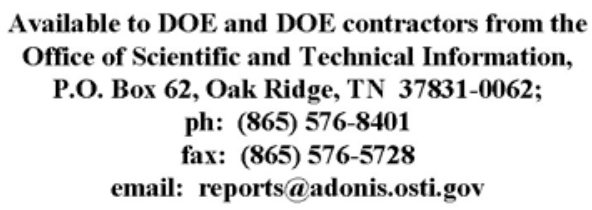

Available to the public from the National Technical Information Service 5301 Shawnee Rd., Alexandria, VA 22312 ph: (800) 553-NTIS (6847) email: orders@ntis.gov $<$ http://www.ntis.gov/about/form.aspx > Online ordering: http://www.ntis.gov 


\section{Rayleigh-Taylor Instability within Sediment Layers Due to Gas Retention: Preliminary Theory and Experiments}

PA Gauglitz

BE Wells

SD Rassat

WC Buchmiller

March 2013

Prepared for

the U.S. Department of Energy

under Contract DE-AC05-76RL01830

Pacific Northwest National Laboratory

Richland, Washington 99352 



\section{Executive Summary}

In Hanford underground waste storage tanks, a typical waste configuration is settled beds of solid particles beneath liquid layers. The settled beds are typically also composed of layers, and these layers can have different physical and chemical properties. One postulated configuration within the settled bed is more-dense layer lying atop a less-dense layer. The different densities can be a result of different gas retention in the layers (Meacham and Kirch 2013) or different degrees of settling and compaction in the layers. This configuration can experience a Rayleigh-Taylor (RT) instability, in which the less-dense lower layer rises into the upper layer. Previous studies of gas retention and release have not considered potential buoyant motion within a settled bed of solids.

The purpose of this report is to provide a review of the literature on RT instabilities with an emphasis on studies that are pertinent to RT instabilities between two solid materials of different bulk density that are formed from settled slurries. A preliminary evaluation of the potential for different gas retention in a sediment layer and gas release from the potential buoyant motion are also discussed, and the results of preliminary experiments that demonstrate RT instabilities due to gas retention are summarized.

Several studies provide useful theoretical and experimental results that can be used to estimate the difference in void fraction (or in bulk density) between two layers that will result in instability within tanks, as well as the effects of yield stress and tank diameter. For soft solids (such as waste slurries), experiments have only been conducted with a slurry layer above a gas layer; experiments with two solid layers will be needed to support the stability criterion developed from the literature data. Evaluations of configurations with more than two layers that have different bulk densities were not available in the literature; however, configurations with multiple-layer systems are expected to be more stable than two layers with the same total difference in bulk density.

Measurements of gas void fractions in slurries in Hanford double-shell waste tanks, when combined with density measurements of de-gassed samples, identified examples in which the gas void fraction increases with depth, and the bulk density (including gas) decreases with depth, showing that that there is a potential for buoyant motion within waste sediment layers arising from differences in bulk density.

Observations of bubble retention in experiments that had gas release pathways, which were generally filled with gas, did not show a noticeable difference in gas retention above and below the depth of an open gas channel. This suggests that gas retention may not necessarily increase below the maximum channel depth. Additional experiments would be required to confirm or refute this observation.

An increase in yield stress (i.e., a stronger material) would be expected to decrease the amount of gas release caused by buoyant motion within a sediment layer, as is the case for buoyant sediment motion into a supernatant liquid, and strong sediment layers may release little gas with buoyant motion, but there are no studies in the literature to support this expectation. This anticipated behavior could potentially be quantified with experiments and modeling.

Two scoping experiments were conducted with kaolin clay slurries. The first experiment used a kaolin slurry with a shear strength of about $15 \mathrm{~Pa}$ and the second experiment used a slurry with a shear strength of about 32 Pa. Each experiment had two horizontal kaolin layers; hydrogen peroxide was added to the lower layer to generate gas bubbles, and thus reduce the bulk density, over the one-day duration of 
the experiment. Both experiments demonstrated buoyant motion once sufficient gas was retained to initiate an instability. The gas void fraction for the onset of the instability increased with increasing shear strength, and the void fraction values at the onset of instability roughly agree with the stability criterion for an RT instability. For both experiments, the amount of gas release was small. 


\section{Acknowledgments}

The authors would like to thank Joe Meacham and Nick Kirch of Washington River Protection Services for helpful technical discussions on this topic area. The authors would also like to thank Dave Rector and Sarah Suffield for careful technical reviews and Maura Zimmerschied for her technical editing. 



\section{Acronyms and Abbreviations}

DST

ET

PNNL

RT

SST

WRPS double-shell tank

elapsed time

Pacific Northwest National Laboratory

Rayleigh-Taylor

single-shell tank

Washington River Protection Solutions 



\section{Contents}

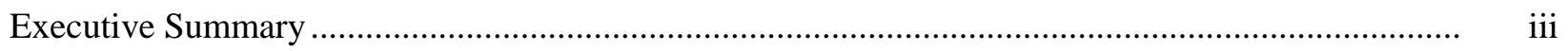

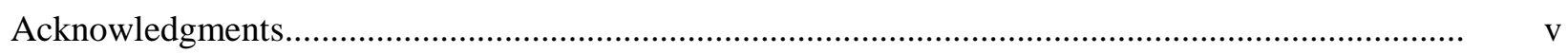

Acronyms and Abbreviations .......................................................................................................... vii

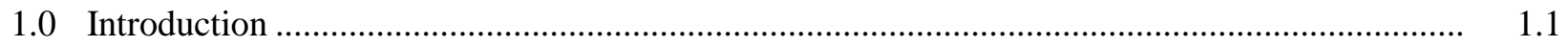

2.0 Review of Rayleigh-Taylor Instability ....................................................................... 2.1

3.0 Potential for Rayleigh-Taylor Instabilities and Gas Release in Settled Waste Layers ................ 3.1

3.1 Potential for Waste Layers with Different Gas Retention and Bulk Density ...................... 3.1

3.2 Examples of Waste Layers with Different Gas Retention and Bulk Density in Hanford Tanks...................................................................................................... 3.2

3.3 Potential for Gas Release from Buoyant Sediment Motion ..............................................

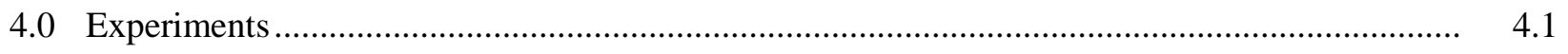

4.1 Experimental Method and Materials ....................................................................... 4.1

4.2 Scoping Test Results .............................................................................................. 4.4

4.2.1 Scoping Test 1: 15 Pa Shear Strength ............................................................... 4.4

4.2.2 Scoping Test 2: 32 Pa Shear Strength ............................................................. 4.9

4.3 Comparison of Test Results to Rayleigh-Taylor Instability Model .................................... 4.14

5.0 Summary and Conclusions ............................................................................................

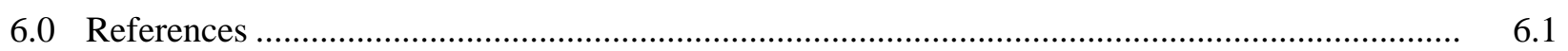




\section{Figures}

1.1 Evolution of a Rayleigh-Taylor Instability of a Less-Dense Waste Layer, due to More Retained Gas Bubbles Rising in a More-Dense Layer

2.1 Void Difference Between Two Layers Needed to Induce a Rayleigh-Taylor Instability in Tanks of Different Diameter Based on Kaolin Clay Properties....................................................

3.1 Slurry Density with Gas for Tank AN-105 ...........................................................................

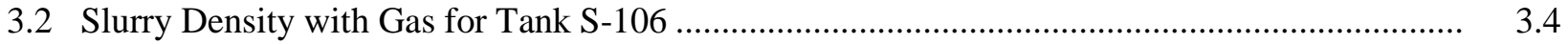

3.3 Slurry Density with Gas for Tank U-103 ............................................................................... 3.4

3.4 Potential Gas Release Scenarios following Buoyancy-Induced Motion within a Sediment Layer.

4.1 Layering of Blue-Dyed Kaolin Slurry with Hydrogen Peroxide, Non-Dyed Kaolin Slurry, and Water in a 10 in. Diameter Vessel in Scoping Tests 1 and 2

4.2 Retained Gas Volume Fraction and the Corresponding Calculated Density of the Bottom Slurry Layer during Scoping Test 1

4.3 Detail of Instability Event in Scoping Test 1: Retained Gas Volume Fraction and the Corresponding Calculated Density of the Bottom Slurry Layer

4.4 Progression of Top and Side Video Camera Images during and Following the Initial Instability Event in Test 1

4.5 Retained Gas Volume Fraction and the Corresponding Calculated Density of the Bottom Slurry Layer during Scoping Test 1

4.6 Instability Event Detail in Scoping Test 2: Retained Gas Volume Fraction and the Corresponding Calculated Density of the Bottom Slurry Layer

4.7 Progression of Top and Side Video Camera Images during and Following the Initial Instability Event in Test 2 .

4.8 Comparison of Model-Predicted Retained Gas Fraction for an RT Instability to the Observed Onset of Instability in the Scoping Tests

\section{Tables}

4.1 Slurry Properties and Initial Conditions in Rayleigh-Taylor Instability Scoping Tests 


\subsection{Introduction}

When two materials of different density are separated at a plane interface, the interface can be unstable when the materials are accelerated in the direction towards the more-dense material. This situation is equivalent to when two materials of different density are under the influence of gravity and the less-dense material is below the more-dense material. This instability is commonly called a Rayleigh-Taylor (RT) instability (Chandrasekhar 1981). The original theoretical study of the instability was conducted by Taylor (1950), and a companion experimental study was reported by Lewis (1950). Chandrasekhar (1981) notes that earlier work by Rayleigh (1883) on the equilibrium of a fluid of variable density under gravitational acceleration is fundamentally the same problem. Many theoretical and experimental studies have been conducted to quantify the behavior of RT instabilities. The purpose of this summary is to provide a review of the literature on RT instabilities with an emphasis on studies that are pertinent to RT instabilities between two solid materials of different bulk density that are formed from settled slurries. A preliminary evaluation of the potential for different gas retention in a sediment layer and gas release from the potential buoyant motion are also discussed, and the results of preliminary experiments that demonstrate RT instabilities due to gas retention are summarized.

In Hanford underground waste storage tanks, a typical waste configuration is settled beds of solid particles beneath liquid layers. The settled beds are typically composed of layers, and these layers can have different physical and chemical properties. One postulated configuration within a settled bed is a less-dense layer beneath a more-dense layer. The different densities can be a result of different gas retention in the layers (Meacham and Kirch 2013) or different degrees of settling and compaction in the layers. Figure 1.1 depicts a configuration of a settled bed with two layers where the lower layer has a lower density than the upper layer due to a larger amount of trapped gas bubbles. This configuration can experience an RT instability, and Figure 1.1 also shows a plausible evolution of the interface between the layers as the less-dense material rises into the upper layer.
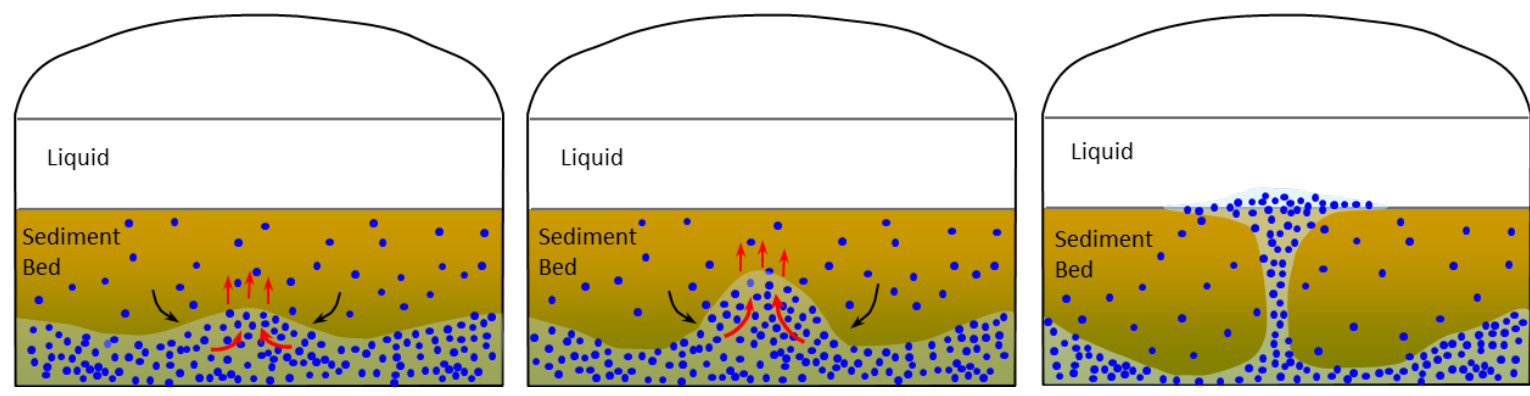

Figure 1.1. Evolution of a Rayleigh-Taylor Instability of a Less-Dense Waste Layer, due to Retained Gas Bubbles (depicted as blue spheres), Rising in a More-Dense Layer

In Hanford waste tanks, a related, but different, buoyancy-driven instability occurs when the sediment bed is less dense than the liquid layer, again due to gas retention in the sediment bed. This instability has been studied extensively (see for example Meyer et al. 1997, Meyer and Stewart 2001, Stewart et al. 2005). These studies include applying theoretical results of RT instabilities in viscous fluids (Meyer et al. 1997), and the overall results have been applied to managing waste storage in the underground tanks (Weber 2009). 



\subsection{Review of Rayleigh-Taylor Instability}

The majority of studies on the RT instability have focused on the behavior of Newtonian fluids. Chandrasekhar (1981) provides a general discussion of the analysis of RT instabilities, including results for inviscid fluids, viscous Newtonian fluids, and the stabilizing effect of surface tension. Detailed reviews on this instability are given by Kull (1991) and Sharp (1984). A typical result from the analysis of the RT instability is an expression for the growth rate of small sinusoidal disturbances, or perturbations, in the interface position for arbitrary wavelengths. The wavelength of the fastest growing disturbance is of particular importance because this represents the most unstable mode. A second useful result is the cutoff wavelength, should one exist, where wavelengths shorter than the cutoff have disturbances that decay with time indicating that the interface is stable for these wavelengths.

While RT instability of fluid/fluid interfaces is well known, and there are a large number of studies reported in the literature for the fluid/fluid problem, the instability also occurs at solid/fluid and solid/solid interfaces but these configurations have received much less attention. For RT instabilities at solid/fluid interfaces, the first theoretical study was given by Miles (1966) and the first experimental study demonstrating the growth of disturbances was presented by Barnes et al. (1974). These initial studies on solid/fluid interfaces showed that the growth of disturbances depends on the dynamic yield strength of the solid and that wavelengths below a critical value are stable. The experiments by Barnes et al. $(1974,1980)$ were conducted using explosions to accelerate metal plates with the acceleration being in the range of $10^{9} \mathrm{~m} / \mathrm{s}^{2}$.

A number of studies have been reported since these original investigations. Terrones (2005) provides a brief review of the available literature on theoretical and experimental studies of RT instabilities at solid/fluid and solid/solid interfaces and other recent summaries are given by Terrones (2007) and Piriz et al. (2005, 2009). The majority of the studies discussed are focused on solid/fluid interfaces. As summarized by both Terrones (2007) and Piriz et al. (2009), the interest in RT instabilities in solid/fluid and solid/solid interfaces includes studies of metals accelerated by gases from high explosives, vaporization of polymers by lasers, ion beam irradiation implosions, and magnetic implosions. For these systems, the accelerations are as high as $10^{11} \mathrm{~m} / \mathrm{s}^{2}$ (Terrones 2005, Piriz et al. 2005).

The first analysis of RT instabilities at solid/solid interfaces was given by Terrones (2005) and additional studies have since been reported by Terrones (2007) and Piriz et al. (2005, 2009). Terrones (2005) gives a particularly useful result which is an analytical relationship for the cutoff wavelength where all disturbances shorter than this wavelength are stable:

$$
\lambda_{c}=\frac{4 \pi\left(G_{1}+G_{2}\right)}{\left(\rho_{2}-\rho_{1}\right) a}
$$

where $G_{1}$ and $G_{2}$ represent the shear moduli for the two materials, $\rho_{1}$ and $\rho_{2}$ are the densities, and $a$ is the

acceleration. Terrones (2005) also provides the relationship for a solid/fluid interface derived by previous authors:

$$
\lambda_{c}=\frac{4 \pi G_{2}}{\left(\rho_{2}-\rho_{1}\right) a}
$$


Both Equations (2.1) and (2.2) show that the cutoff wavelength increases with increasing shear modulus and increases with decreasing density difference. While these studies have been focused on RT instabilities in metal plates and at very high accelerations, the RT instability can also occur in weak solids under the influence of gravitational acceleration.

Epstein (2002) gives the first discussion of the RT instability for a weak solid representative of waste slurries under gravitational acceleration. In this work, a relationship for the onset of the instability that is similar to Equation (2.1) was developed for the configuration of a weak solid above a gas layer of negligible density. Epstein used the original theoretical work of Miles (1966), together with theoretical arguments, to develop an equation for the cutoff wavelength with an unknown constant. He then used the original experimental results from Barnes et al. (1974) for plate accelerations of $\sim 10^{9} \mathrm{~m} / \mathrm{s}^{2}$ to estimate the constant. The result of this analysis was the following:

$$
\lambda_{c}=\frac{12 \pi \tau_{2}}{\rho_{2} g}
$$

where $\tau_{2}$ is the yield stress and $\rho_{2}$ is the density of the weak solid (in subsequent use of this result, shear-strength values from vane measurements were used for $\tau_{2}$ ). Note that the form of Equation (2.3) is equivalent to that of Equations (2.1) and (2.2) from Terrones (2005) with a different constant and using the yield stress rather than the shear modulus to represent the mechanical properties of the solid. Epstein (2002) then assumed that the longest-wavelength disturbance that could grow in a cylindrical vessel could be estimated as the vessel diameter $D$, so $\lambda_{c} \sim D$, giving the following stability criterion by rearranging Equation (2.3):

$$
Y_{G}=\frac{\tau}{\rho_{2} g D}=\frac{1}{12 \pi}
$$

The dimensionless group in Equation (2.4) is commonly called the gravity yield parameter $Y_{G}$ and the stability criterion is $Y_{G}=1 / 12 \pi=0.027$. For $Y_{G}$ below the criterion a configuration is unstable, and at $Y_{G}$ values above the criterion the configuration is stable.

Epstein and Gauglitz (2010) tested the validity of Equation (2.4) with a series of experiments using kaolin clay slurries with shear-strength values ranging from 38 to $1600 \mathrm{~Pa}$ and cylindrical vessels with diameters ranging from 1.7 to $45 \mathrm{~cm}$. This study also used two different experimental methods. The experimental results supported the scaling given by the dimensionless gravity yield parameter, and the experimental value for the criterion was the following:

$$
Y_{G}=0.09
$$

Epstein and Gauglitz (2010) reported additional results for vessel geometries that were not cylindrical and Gauglitz et al. (2010) summarizes studies with additional simulants and a range of layer depths.

The result from Terrones (2005) given by Equation (2.1) can be written in form similar to Equation (2.4) and used as a criterion for instability in systems where both the upper and lower regions are solids and the acceleration is due to gravity:

$$
\frac{\left(G_{1}+G_{2}\right)}{\left(\rho_{2}-\rho_{1}\right) g D}=\frac{1}{4 \pi}
$$


This result uses the shear moduli of the elastic materials rather than the yield stress used in Equation (2.4). Wells et al. (2010a, 2010b) have reported shear-modulus and shear-strength results for two simulants designed to mimic Hanford waste. The relationship between shear modulus and shear strength was also provided for kaolin and bentonite clay simulants. For these simulant materials, the shear modulus was higher than the shear strength and the magnitude of the difference, which varied from about 2 to 1200, depended on the method used for making the measurements and the simulant (Wells et al. 2010b). For the purpose of modifying Equation (2.6), the shear moduli in Equation (2.6) will be estimated as $G_{i} \sim c \tau_{i}$, where $\tau_{i}$ is the shear strength and $c$ is an unknown constant, giving the following result for the stability criterion for two solid layers:

$$
Y_{G}=\frac{\left(\tau_{1}+\tau_{2}\right)}{\left(\rho_{2}-\rho_{1}\right) g D}=\frac{1}{4 \pi c}
$$

The constant for the stability criterion is, again, a theoretical result from the linear stability analysis for infinitesimal disturbances together with an unknown constant $c$. The experimental result from Epstein and Gauglitz (2010) given by Equation (2.5), even though it was measured for a solid/fluid interface, is probably a better estimate for the expected value of $Y_{G}$ in cylindrical vessels. This assumes that the experimental value for the constant defining the stability boundary with a solid/fluid interface will be similar to a solid/solid interface when the strength of both layers is included as given by Equation 7 . Using this experimental result, the stability criterion for an RT instability with two solid layers of different density becomes the following:

$$
\frac{\left(\tau_{1}+\tau_{2}\right)}{\left(\rho_{2}-\rho_{1}\right) g D}=0.09
$$

where $\tau_{1}$ and $\tau_{2}$ are the shear strengths of the two layers. The density difference can be represented in terms of gas void fraction, assuming equal initial densities for the slurry in both layers 1 and 2, as follows:

$$
\left(\rho_{2}-\rho_{1}\right)=\Delta \alpha \rho_{s}
$$

where $\Delta \alpha$ is the gas void fraction difference and $\rho_{s}$ is the slurry density, which is constant in both sediment layers. The stability criterion (Equation (2.8)) together with Equation (2.9) gives the conditions of the onset of an RT instability as a function of the void fraction (difference), the strength and density of the sediment layers, and the vessel diameter.

Figure 2.1 shows the result of using the stability criterion given by Equation (2.8), assuming the strengths of the two layers are the same, together with shear strength and density values for kaolin clay slurries given in Gauglitz et al. (2012). The different lines represent three smaller vessels that are suitable for small-scale experiments $(5,10$, and $23 \mathrm{in}$. diameter $)(0.127,0.254$, and $0.584 \mathrm{~m})$ and a full-scale double-shell tank (DST) with a diameter of $22.9 \mathrm{~m} \mathrm{(75} \mathrm{ft).} \mathrm{These} \mathrm{results} \mathrm{show} \mathrm{that} \mathrm{the} \mathrm{void} \mathrm{difference}$ needed to initiate an RT instability increases with increasing strength. The results also show that, for a given shear strength, the void increase needed to initiate an RT instability decreases with increasing tank diameter. Figure 2.1 also shows the range of void fraction that can typically be reached in clay simulants as a function of shear strength (Rassat et al. 1998, Gauglitz et al. 2012). In the shaded region, bubble release occurs and a void fraction that high cannot be achieved. The evaluation shows that the smallest vessel shown ( $0.127 \mathrm{~m}$ or $5 \mathrm{in}$.) will only give an RT instability in lower shear-strength material, but the material is essentially too weak to retain sufficient gas void fraction to initiate the instability. However, 
experimental tests in $0.254 \mathrm{~m}$ (10 in.) and larger vessels should be able to demonstrate RT instabilities and gas void fractions that can be achieved.

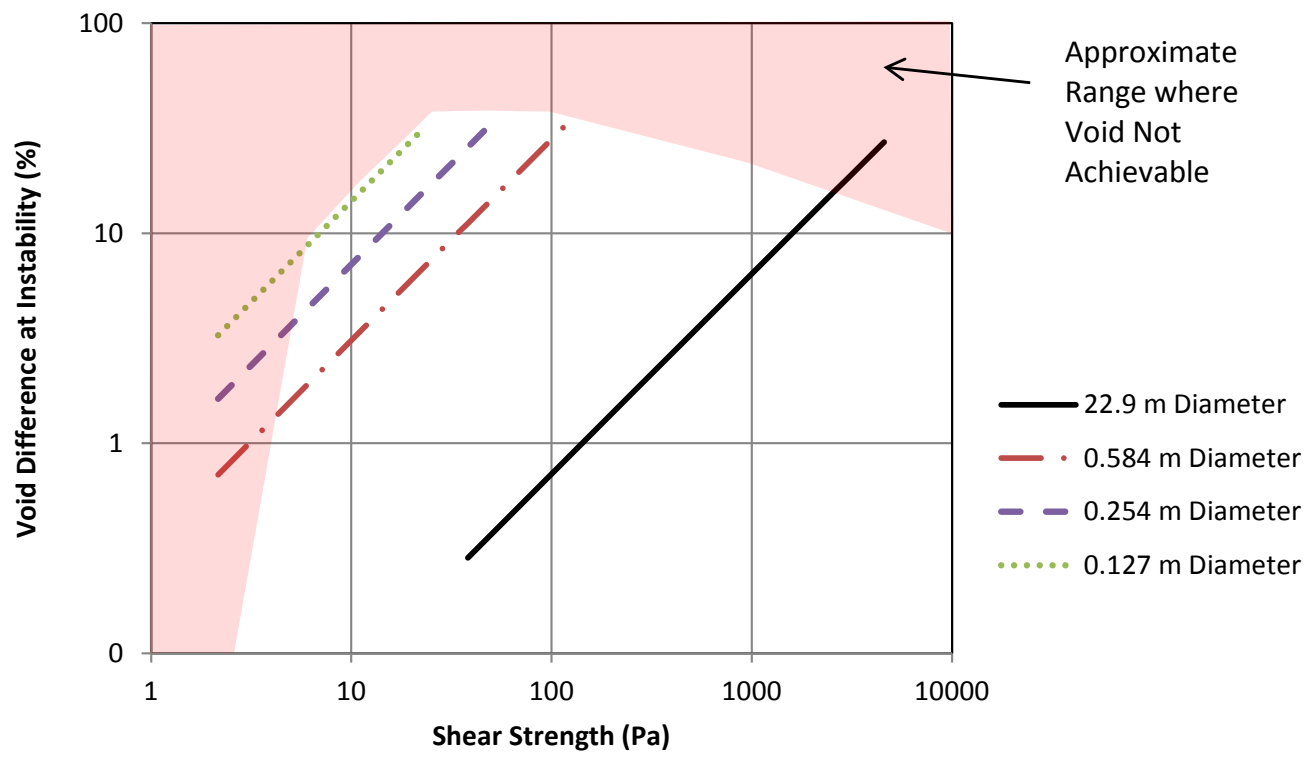

Figure 2.1. Void Difference Between Two Layers Needed to Induce a Rayleigh-Taylor Instability in Tanks of Different Diameter Based on Kaolin Clay Properties (the shaded region indicates gas retention values that are higher than can be achieved in experiments.)

The configuration and stability results for two distinct layers are a simplification of the complicated layering that probably occurs in actual tank sediment. The stability criterion shows that smaller density differences (or smaller void differences) tend to make a configuration stable. Conceptually, an interface with a density difference between two layers that is unstable can be made stable by having three layers where the interface between each pair of layers is stable. The literature does not evaluate configurations with multiple layers of different bulk density, and configurations with multiple-layer systems are expected to be more stable than two layers with the same total difference in density as the multiple-layer system. 


\subsection{Potential for Rayleigh-Taylor Instabilities and Gas Release in Settled Waste Layers}

For an RT instability to occur within a waste sediment bed there must be a sufficient difference in the bulk densities of two layers for a lower buoyant layer to overcome the waste strength for the longest wavelength that can occur in the sediment bed. The bulk density includes the contribution from retained gas. As discussed by Meacham and Kirch (2013), one postulated scenario is having a higher gas retention in a lower layer and thus a lower bulk density than a region above (see Figure 1.1). A brief assessment of the potential for this postulated configuration is given below.

Should a waste layer within a sediment bed be unstable and begin to rise as shown in Figure 1.1, some fraction of the retained gas will probably be released. The magnitude of the release is likely to depend on the waste properties, in particular the rheology of both the buoyant lower layer and the upper layer. A brief assessment of the physical mechanisms affecting the gas release is also given below.

\subsection{Potential for Waste Layers with Different Gas Retention and Bulk Density}

The postulated mechanism for having higher gas retention in a lower layer is based on an evaluation of channel formation in sediment layers when gas generation is occurring. The channels are described as pathways filled with water and gas bubbles that are formed during consolidation of the sediment bed together with gas generation, and the channels have a maximum depth. Van Kessel and Van Kesteren (2002) have summarized observations of a single large-scale test of bubble retention in lake-bottom sediment taken from Ketelmeer in the Netherlands where these channels were observed and have also summarized the development of a model for the observed behavior. Winterwerp and van Kesteren (2004) give an additional summary of these observations and mechanisms, details of the model development are given by Van Kessel (1999) and Crosato (1998), and Meacham (2010) has discussed how to apply these concepts to Hanford tank waste.

Van Kessel (1999) specifically comments that below the maximum channel depth the channels do not contribute to gas release. Van Kessel (1999) further comments that the release of gas from below the maximum channel depth will only occur when the sediment becomes unstable. Van Kessel and Van Kesteren (2002) estimated that the maximum channel depth in the Ketelmeer sediment is about 8-10 m. The large-scale test with Ketelmeer sediment had about $5 \mathrm{~m}$ of sediment, so this test could not show a difference in bubble retention behavior above and below the maximum channel depth. For comparison, Meacham and Kirch (2013) have noted that the estimated maximum channel depth for DST waste sludges is between 4.4 and $9.8 \mathrm{~m}$. To date, no experiments have been conducted that are deep enough to observe the potential difference above and below the maximum channel depth.

Gauglitz et al. (2012) studied gas retention behavior in kaolin clay simulants and conducted tests with sediment depths up to $1.5 \mathrm{~m}$. In these tests, the clay simulants had very little consolidation during the time period of gas generation. Accordingly, in contrast to the Ketelmeer sediment tests (Van Kessel and Van Kesteren 2002) in which water removal was a factor in channel formation, the kaolin clay tests did not have water release during consolidation to contribute to channel formation. In the kaolin clay tests (Gauglitz et al. 2012), channels were observed as primary pathways for gas release and the channels were 
gas-filled. Along these gas-release pathways, there were locations where water would block the movement of gas and periodically the gas would displace the water blockage and flow..

For the gas-filled channels observed by Gauglitz et al. (2012), it is not clear if the model for the maximum channel depth given by Van Kessel and Van Kesteren (2002), which assumes a hydrostatic pressure from water within the channel, should represent the channel behavior. In a separate study, Gauglitz et al. (2010) have conducted tests to measure the depth of open gas channels for different simulants, and the measured depth was $12 \mathrm{~cm}$ for $55 \mathrm{wt} \%$ kaolin. For the bubble retention tests where gas-filled channels were observed, the kaolin concentration was $55 \mathrm{wt} \%$, the shear strength was $680 \mathrm{~Pa}$, and the initial sediment depth was $0.8 \mathrm{~m}$ (Gauglitz et al. 2012). Accordingly, the test with an initial sediment depth of $0.8 \mathrm{~m}$ was much deeper than the measured depth of an open gas channel for this simulant. In the observations of gas retention (Gauglitz et al. 2012), there was no apparent difference in bubble retention above and below the depth corresponding to the open-channel depth. What was observed was periodic motion of gas bubbles along pathways that became established and were channels for gas release. The established pathways appeared to extend below what can be estimated to be the maximum depth of an open gas channel.

In summary, the observations of Gauglitz et al. (2012) show channel formation for gas release pathways, in agreement with Van Kessel and Van Kesteren (2002). The results show similar gas retention above and below the depth of an open gas channel, but it is not yet clear if this measure depth is appropriate for evaluation of the observed bubble retention behavior. Additional studies might confirm or refute if the postulated increase in gas retention below a maximum channel depth does not occur.

\subsection{Examples of Waste Layers with Different Gas Retention and Bulk Density in Hanford Tanks}

While the observations of Gauglitz et al. (2012) suggest that bubble retention is the same above and below the maximum channel depth, other factors may cause gas retention to vary with depth in a sediment layer. As an example, the in situ gas void fraction was measured in a number of the Hanford tanks, and the void fraction increases with depth (tanks SY-103 and AN-104 are examples) for a number of tanks (Stewart et al. 1996a).

Data are available for gas void fraction and slurry density (degassed) for a number of DSTs and single-shell tanks (SSTs). These data were used with the following equation to estimate the bulk density (including the retained gas) as a function of depth for selected tanks:

$$
\rho_{b}=(1-\alpha) \rho_{s}
$$

where $\rho_{b}$ is the density of the slurry with gas, $\alpha$ is the gas void fraction, and $\rho_{s}$ is the density of the degassed slurry (this assumes the gas density is much lower than degassed slurry density). Figure 3.1 and Figure 3.2 show the estimated slurry density with gas for the sediments in DST AN-105 and SST S-106. The slurry density with gas estimates are made using simple linear and polynomial fits to the degassed slurry density and gas void fraction with depth. AN-105 has a deep supernatant liquid layer, has exhibited large gas release events (Hedengren et al. 2000), and is shown to have decreasing density with depth, which is a potentially unstable configuration. S-106 did not have a supernatant layer, has exhibited no gas release events (Hedengren et al. 2001), and is shown to have increasing density with depth, which 
is a stable configuration. Conversely, SST U-103, also with no supernatant layer, has had numerous gas release events (Hedengren et al. 2001), but the estimated slurry density with gas also increases or is relatively constant with depth, which is a stable configuration, as shown in Figure 3.3. Thus, tank farm data demonstrate that there is a potential for instabilities due to density differences, but there may be no clear correlation between the potential for RT instabilities and the observed gas release behavior.

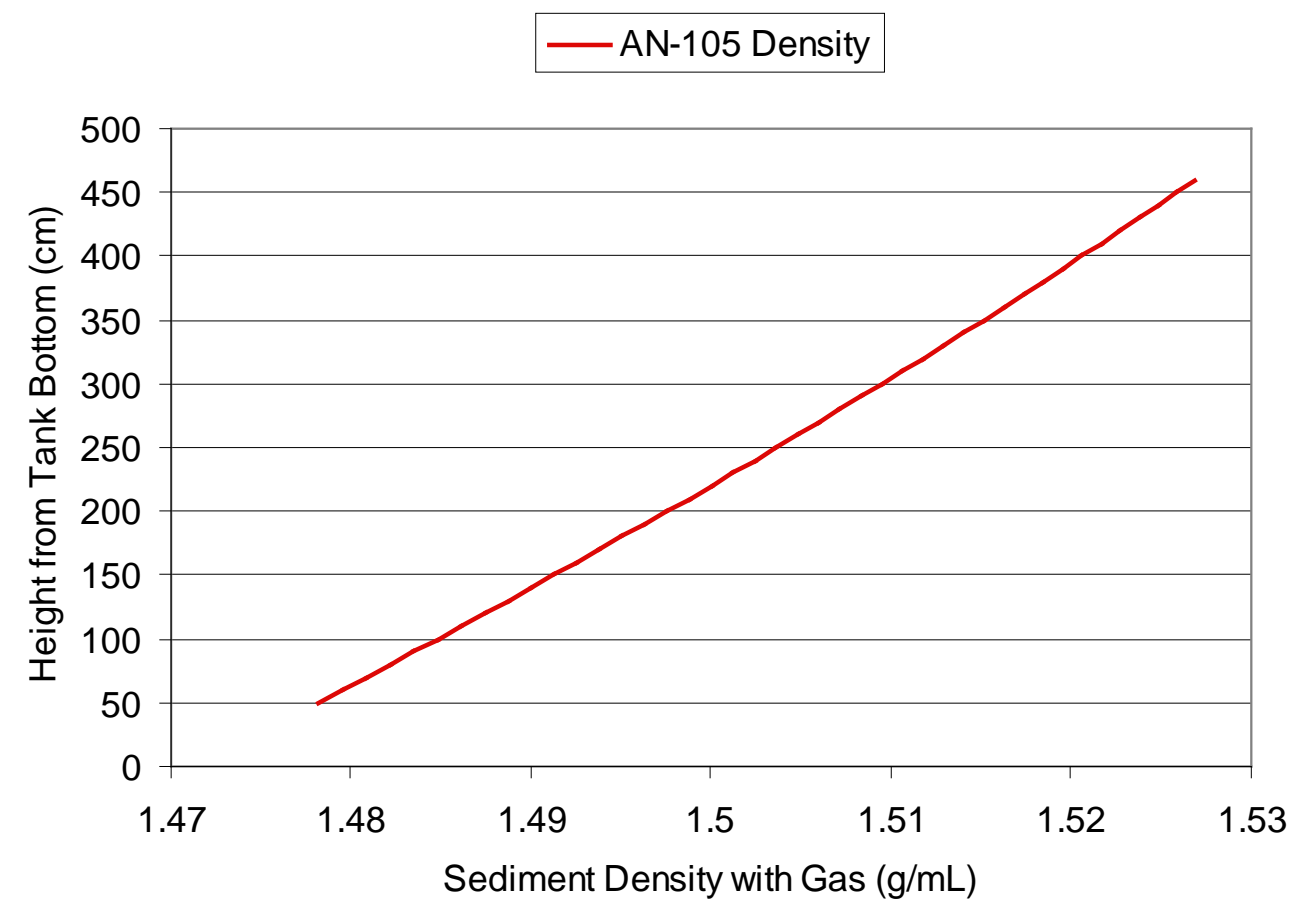

Figure 3.1. Slurry Density with Gas for Tank AN-105 


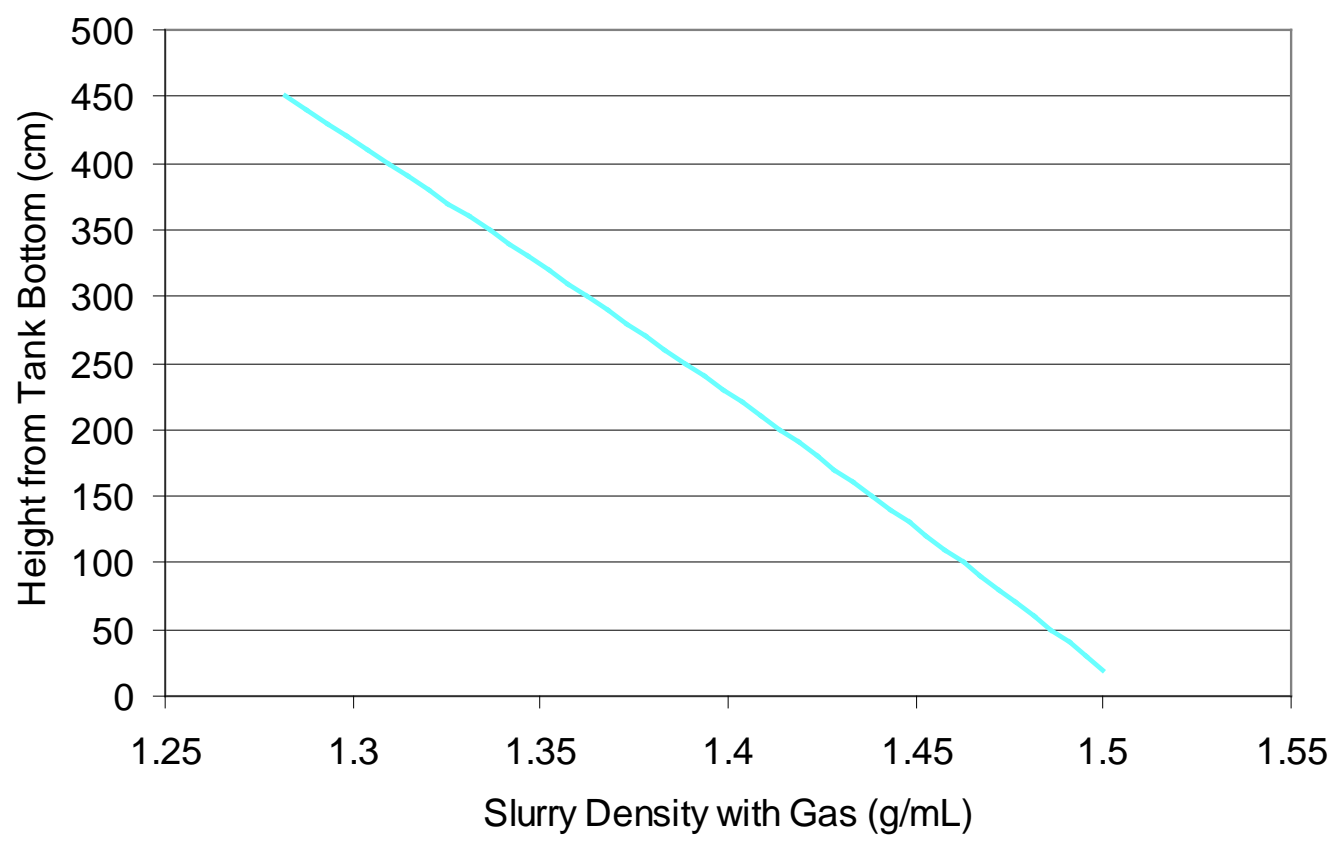

Figure 3.2. Slurry Density with Gas for Tank S-106

\section{U-103}

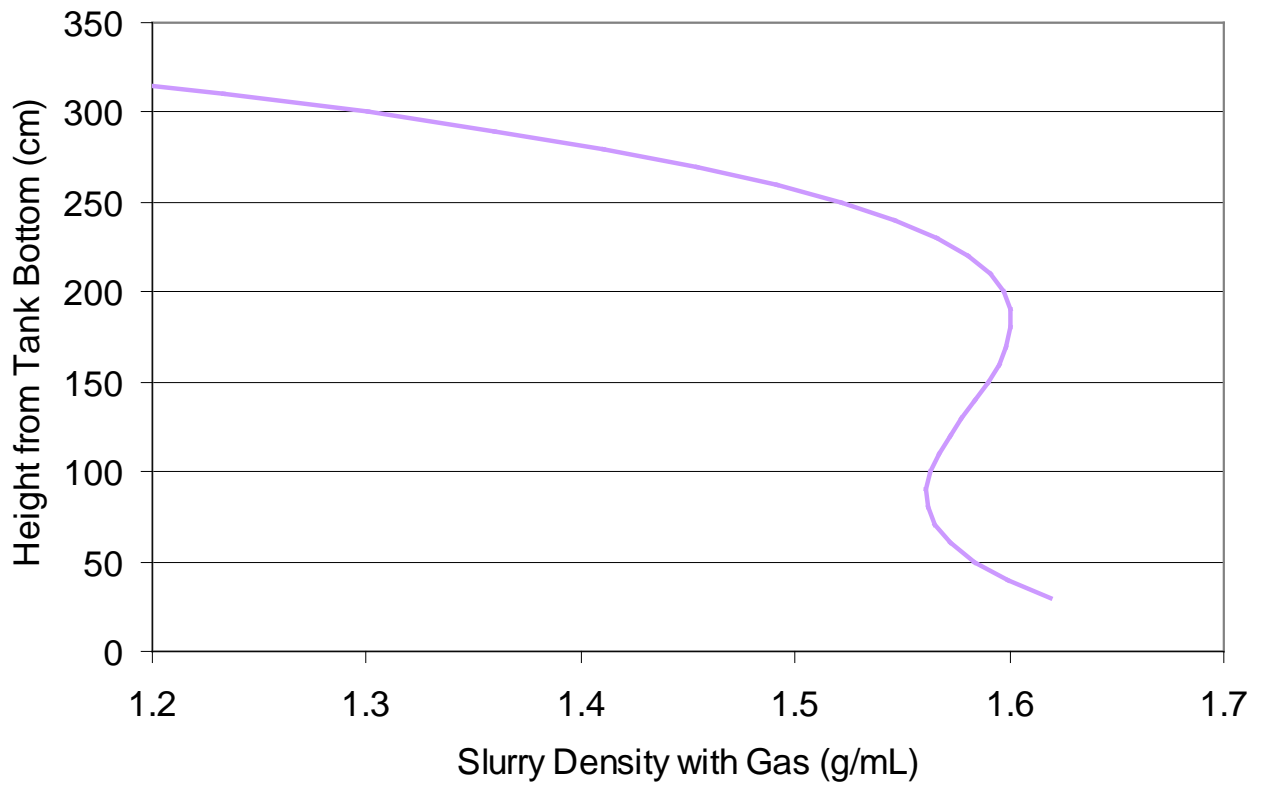

Figure 3.3. Slurry Density with Gas for Tank U-103 


\subsection{Potential for Gas Release from Buoyant Sediment Motion}

If an RT instability were to occur within a sediment layer, the motion of a lower buoyant layer may allow some of the retained gas bubbles to move through the sediment and be released. It is also possible that the buoyant layer may retain sufficient gas that sediment is buoyant in the liquid layer above the sediment. In this situation, the buoyant motion through the liquid layer will also contribute to the release of retained gas. Both of these scenarios are depicted in Figure 3.4.
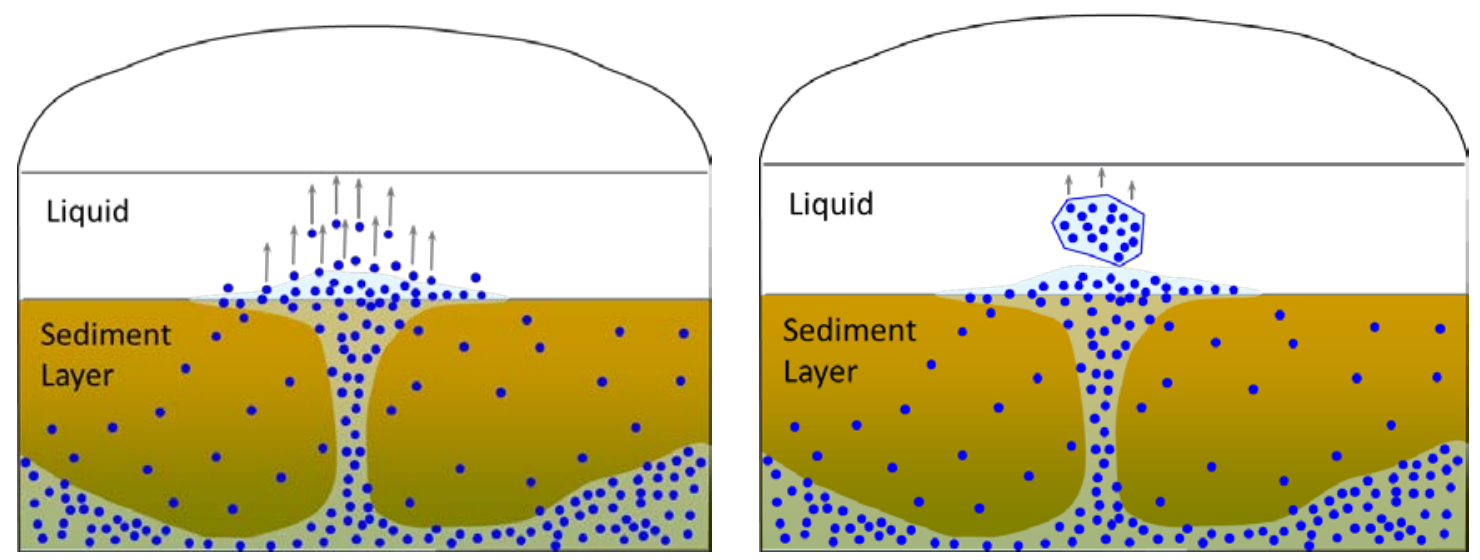

Figure 3.4. Potential Gas Release Scenarios following Buoyancy-Induced Motion within a Sediment Layer

The amount of gas that can be released from the induced motion is likely to depend on the shear strength of the material and the buoyant potential energy available to shear the bubbly sediment. Previous studies have observed these effects for sediment layers that are buoyant in the liquid above (Stewart et al. 1996b) and models have been developed to quantify the amount of sediment shearing, and hence gas release, that occurs in different configurations (Meyer et al. 1997, Meyer and Stewart 2001). While the magnitude of gas release from the potential buoyant motion within a sediment layer should decrease with increasing yield stress, there are no data to support this anticipated behavior. 



\subsection{Experiments}

Hanford waste storage tanks have large diameters (typically $75 \mathrm{ft}, 22.9 \mathrm{~m}$ ) and often contain strong sludge (e.g., 1000 Pa shear strength)(Meacham 2010). Scoping experiments with relatively weak kaolin clay slurries were conducted so that the postulated RT instability resulting from a lower-density and higher gas-fraction waste stored beneath a higher density waste could be investigated in a small, laboratory bench-scale vessel (see Equation (2.8)). In this section, the experimental method and materials and the test conditions used in two RT instability scoping experiments are presented. The results of these tests are then described in detail; finally, the results are compared to model predictions of the RT instability.

\subsection{Experimental Method and Materials}

Two RT instability scoping experiments were completed using kaolin clay-deionized water slurries of different solids mass fractions, shear strengths, and bulk densities. In each scoping test, a bottom layer of blue-dyed slurry containing a small mass fraction of liquid hydrogen peroxide $\left(\mathrm{H}_{2} \mathrm{O}_{2}\right)$ to generate oxygen $\left(\mathrm{O}_{2}\right)$ gas (and water) was covered by a middle layer of naturally colored kaolin slurry and a top layer of supernatant water. Initially, the two slurry layers had nominally identical solids mass fraction, shear strength, and density. It was planned that the density of the bottom layer would be reduced during the course of the experiment as a result of $\mathrm{H}_{2} \mathrm{O}_{2}$ decomposition and the retention of $\mathrm{O}_{2}$ gas bubbles (voids). The kaolin slurries in each scoping test were prepared similarly. Kaolin and water were mixed by hand or with a power mixer late the afternoon before the test, allowing the kaolin to hydrate overnight. Water was withheld from the premixed dyed-kaolin slurry so that the required amount of $3 \mathrm{wt} \% \mathrm{H}_{2} \mathrm{O}_{2}$ solution and its bulk water could be added just before the experiment, bringing the slurry to the target kaolin mass fraction. Both the dyed and natural kaolin slurries were remixed prior to loading in the test vessel. A concentrated form of blue dye was used, and its mass fraction in the slurry, $<0.1 \mathrm{wt} \%$, was effectively negligible.

Bulk density of the slurries was calculated (estimated) from the masses (or mass fractions) and densities of individual slurry components, as described, for example, in Gauglitz et al. (2010) for kaolin slurries. Consistent with this previous work, a kaolin solid density of $2.65 \mathrm{~kg} / \mathrm{L}\left(2650 \mathrm{~kg} / \mathrm{m}^{3}\right)$ and a room-temperature water density of $0.998 \mathrm{~kg} / \mathrm{L}\left(998 \mathrm{~kg} / \mathrm{m}^{3}\right)$ were used in the calculation of bulk density. The shear strength of the kaolin slurry was estimated, as a function of kaolin mass fraction, from a previously established correlation (see the equation in Figure 3.1 of Gauglitz et al. (2010)). The mass fraction of kaolin and the calculated slurry density and shear strength in both slurry layers of the two scoping tests are shown in Table 4.1. The nominal values of these parameters in Test 1 were $35.0 \mathrm{wt} \%$, $1.28 \mathrm{~kg} / \mathrm{L}\left(1280 \mathrm{~kg} / \mathrm{m}^{3}\right)$, and $15 \mathrm{~Pa}$, respectively; in Test 2 they were $39.0 \mathrm{wt} \%, 1.32 \mathrm{~kg} / \mathrm{L}\left(1320 \mathrm{~kg} / \mathrm{m}^{3}\right)$, and $32 \mathrm{~Pa}$.

Slurry layers and a supernatant water layer were loaded in a 10 in. (0.254 m) inside-diameter vessel. The clear plastic, flat-bottom vessel (without fins) is identical to that used by Gauglitz et al. (2010). A clear plastic ruler with $1 \mathrm{~mm}$ demarcations was affixed to the outside of the vessel and zeroed at the internal bottom of the vessel. The vessel was loaded in the following manner. The blue-dyed slurry was added to the bottom of the vessel and settled/smoothed to provide a nominally flat surface, at which point the slurry level was measured and recorded. The second natural-kaolin slurry layer was added to a depth approximately equal to that of the first layer, taking care to minimize disturbance of the bottom dyed 
slurry. The total depth of slurry after flattening was again noted. Finally, the supernatant water was added and the total depth recorded. The depth of each layer was obtained by difference of the totals recorded during the filling process, and the volume of each layer was estimated from the measured layer depths and the calculated vessel cross-section. The layer depths and volumes are shown in Table 4.1. Slurry depths ranged from 13 to $15 \mathrm{~cm}(0.13$ to $0.15 \mathrm{~m})$, the water depth was 8 to $9 \mathrm{~cm}(0.08$ to $0.09 \mathrm{~m})$, and the total depth was consistently $36 \mathrm{~cm}(0.36 \mathrm{~m})$.

Table 4.1. Slurry Properties and Initial Conditions in Rayleigh-Taylor Instability Scoping Tests

\begin{tabular}{llcc}
\hline \multicolumn{1}{c}{ Property or Test Condition } & \multicolumn{1}{c}{ Layer } & Test 1 & Test 2 \\
\hline Mass Fraction Kaolin (wt\%) & Bottom, Dyed Kaolin & 35.0 & 39.0 \\
& Middle, Natural Kaolin & 35.0 & 39.0 \\
Calculated Shear Strength (Pa) & Bottom, Dyed Kaolin & 15 & 32 \\
& Middle, Natural Kaolin & 15 & 32 \\
\hline Slurry Density (kg/L) & Bottom, Dyed Kaolin & 1.279 & 1.323 \\
& Middle, Natural Kaolin & 1.277 & 1.319 \\
\hline Layer Depth (cm) / Volume (L) & Bottom, Dyed Kaolin & $13.5 / 6.8$ & $14.1 / 7.1$ \\
& Middle, Natural Kaolin & $14.5 / 7.4$ & $13.0 / 6.6$ \\
& Water & $8.0 / 4.0$ & $8.9 / 4.5$ \\
& Total & $36.0 / 18.2$ & $36.0 / 18.2$ \\
Retained Gas at Neutral Buoyancy in Water (vol\%) & Bottom, Dyed Kaolin & 21.9 & 24.6 \\
Absolute Pressure at Mid-depth (atm) & Bottom, Dyed Kaolin & 1.034 & 1.034 \\
\hline
\end{tabular}

Figure 4.1 shows the filled vessel near the beginning of the scoping tests. The photo of Test 1 in Figure 4.1 (left) is indicative of the difficulty in layering the relatively weak slurry ( 15 Pa shear strength). Addition of the natural-kaolin slurry significantly disturbed the originally flat dyed-kaolin slurry, despite the care taken to prevent such. This is evidenced by the streaking of blue clay up into the natural clay layer along the vessel wall. In part due to the nonuniformity in the slurry interface, retained gas volumes during the experiments were determined by tracking the water surface level.

Tripod-mounted digital video cameras (Edmund Optics, EO-1918C CCD) were located to one side and above the top of the vessel, and images were recorded at one frame per second throughout the tests. The side-view camera was positioned to read the affixed ruler. Level data were taken from these images post-experimentally, typically at a $1 \mathrm{~mm}$ resolution, corresponding to $\sim 0.7 \mathrm{vol} \%$ increments in retained gas based on the initial volume of the bottom slurry layer,.

The absolute pressure at mid-depth of the bottom layer, $\sim 1.034 \mathrm{~atm}\left(\sim 1.048 \times 10^{5} \mathrm{~Pa}\right)$ in both tests, was calculated from layer depths and densities, assuming an atmospheric pressure of $1.0 \mathrm{~atm}(1.01325 \times$ $10^{5} \mathrm{~Pa}$ ). The difference in pressure from the mid-depth of the bottom layer to the water surface at atmospheric pressure is indicative of the average gas volume expansion, $\sim 3.4 \mathrm{vol} \%$, should bubbles rise to the water surface. Also of interest in assessment of potential gas release mechanisms is the retained gas volume fraction in the slurry required for it to become neutrally buoyant in the supernatant liquid (e.g., water). Neglecting bubble expansion due to the pressure differential, the estimated retained gas fractions of the bottom slurry layers at neutral buoyancy in water are 21.9 and $24.6 \mathrm{vol} \%$ in Tests 1 and 2, respectively. Sufficient hydrogen peroxide was added to the slurry in the bottom layer of each 
experiment that $>50$ vol\% of $\mathrm{O}_{2}$ could be generated in the layer, if all gas was retained, and therefore, neutral buoyancy in water was possible. The absolute pressure at depth and the gas fraction at neutral buoyancy are also summarized in Table 4.1.
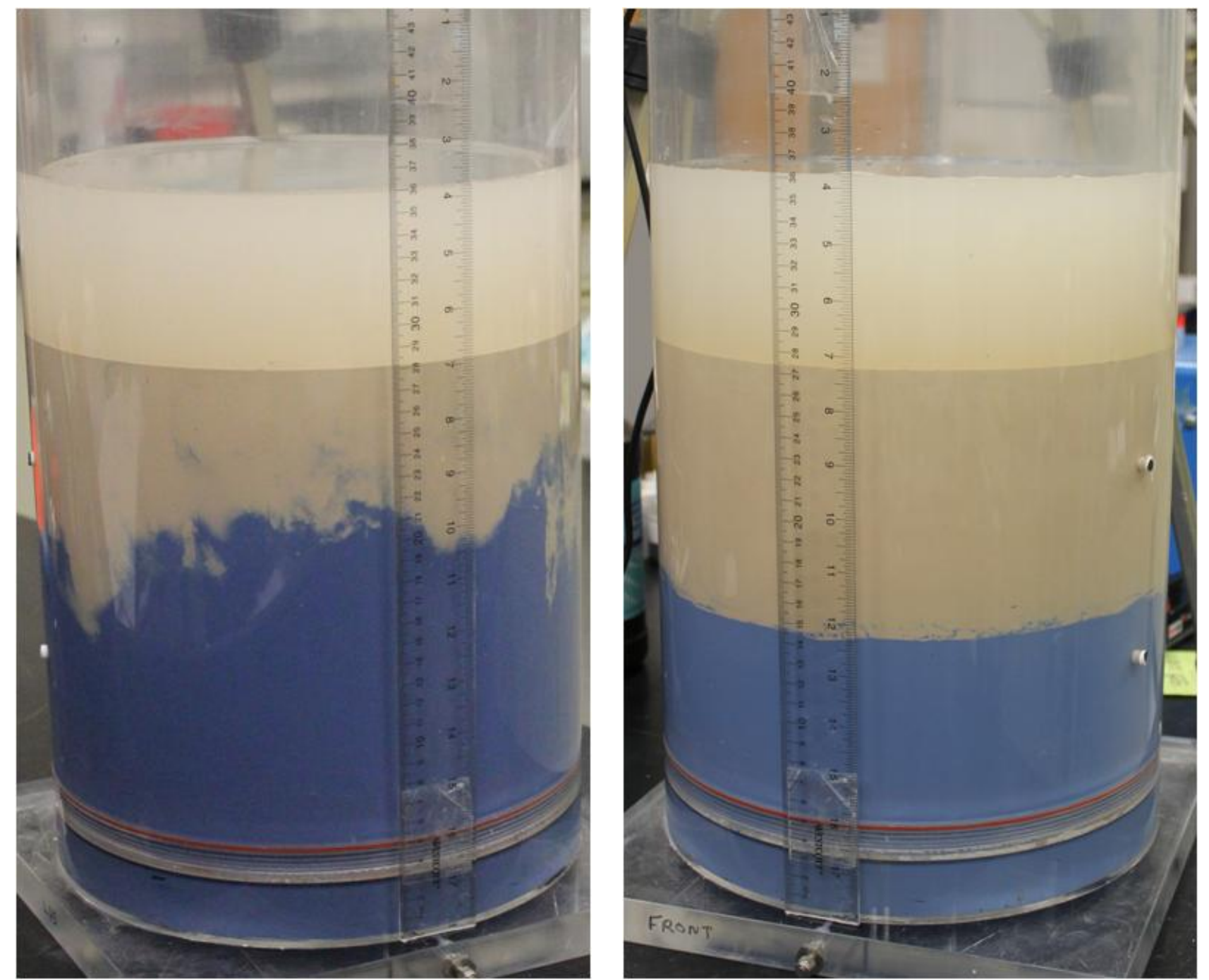

Figure 4.1. Layering of Blue-Dyed Kaolin Slurry with Hydrogen Peroxide (Bottom), Non-Dyed Kaolin Slurry (Middle), and Water in a 10 in. Diameter Vessel in Scoping Tests 1 (Left) and 2 (Right) 


\subsection{Scoping Test Results}

The results of the two RT instability scoping tests using kaolin slurries of different shear strengths are described below. The start of a test, elapsed time (ET) zero, was correlated to the clock time when addition and stabilization of the supernatant water layer was completed. The absolute value of ET is not important in the interpretation of the results, although any retained gas at ET 0 due to decomposition of hydrogen peroxide in the bottom slurry layer from the time of addition would not be accounted for. Considering the relatively slow rate of gas generation, as shown below, the initial retained gas fraction is assumed to be negligible. For these scoping tests, there was about a $30 \mathrm{~min}$ delay between the initial addition of the hydrogen peroxide and the initial water level measurement for tracking the increase in retained gas. The volume of gas retained during an experiment was determined from the change in water level referenced to the condition at the start of the test. In turn, the retained gas volume fraction in the bottom kaolin slurry layer was calculated from the measured gas volume and the initial volume of the dyed slurry. Likewise, the density of the bottom slurry layer was calculated from the initial density and volume of slurry and the measured volume of retained gas (neglecting the mass of $\mathrm{O}_{2}$ gas).

\subsubsection{Scoping Test 1: $\sim 15 \mathrm{~Pa}$ Shear Strength}

The two layers of kaolin slurry in the first scoping test each had an initial shear strength of $\sim 15 \mathrm{~Pa}$ and density of $\sim 1.28 \mathrm{~kg} / \mathrm{L}$, as discussed in Section 4.1 . For this test, the expected density of the kaolin layer at the onset of instability should be less than the water layer, and the expected motion should follow what is shown in the left-hand picture of Figure 3.4. In Figure 4.2, the left axis shows the volume fraction of retained gas in the bottom slurry layer during the test and the right axis shows the slurry density. After an initial lag of $\sim 100 \mathrm{~min}$, the figure shows a nearly linear increase in the volume fraction of retained gas. Movement of the slurry was detected at the vessel wall at an ET of $\sim 370 \mathrm{~min}$ and a retained gas fraction of $\sim 5$ vol\%. The event is marked in Figure 4.2 as the start of the instability. The volume of retained gas remained constant for a period of $\sim 80$ min after the first minute of the instability event, beyond which the volume fraction of retained gas continued to increase almost linearly, as shown in Figure 4.2. The period of constant gas fraction is consistent with a balance between the rate of gas generation and gas release. Additional instability events were recorded, but they are not depicted in Figure 4.2 and are not discussed further, as they are not pertinent to the assessment of the initial RT instability event.

Figure 4.3 provides a more detailed view of the volume fraction of retained gas and slurry density in the period surrounding the initial instability event, and Figure 4.4 shows a progression of top- and sideview video camera images of the event. For convenience, the alphabetic labels and corresponding time markers in the event detail plot, Figure 4.3, are keyed to images shown in Figure 4.4. Note that the ruler affixed to the side of the vessel is at one o'clock in the top-view images. Also, elapsed time zero in these figures is defined as the start of the event. 


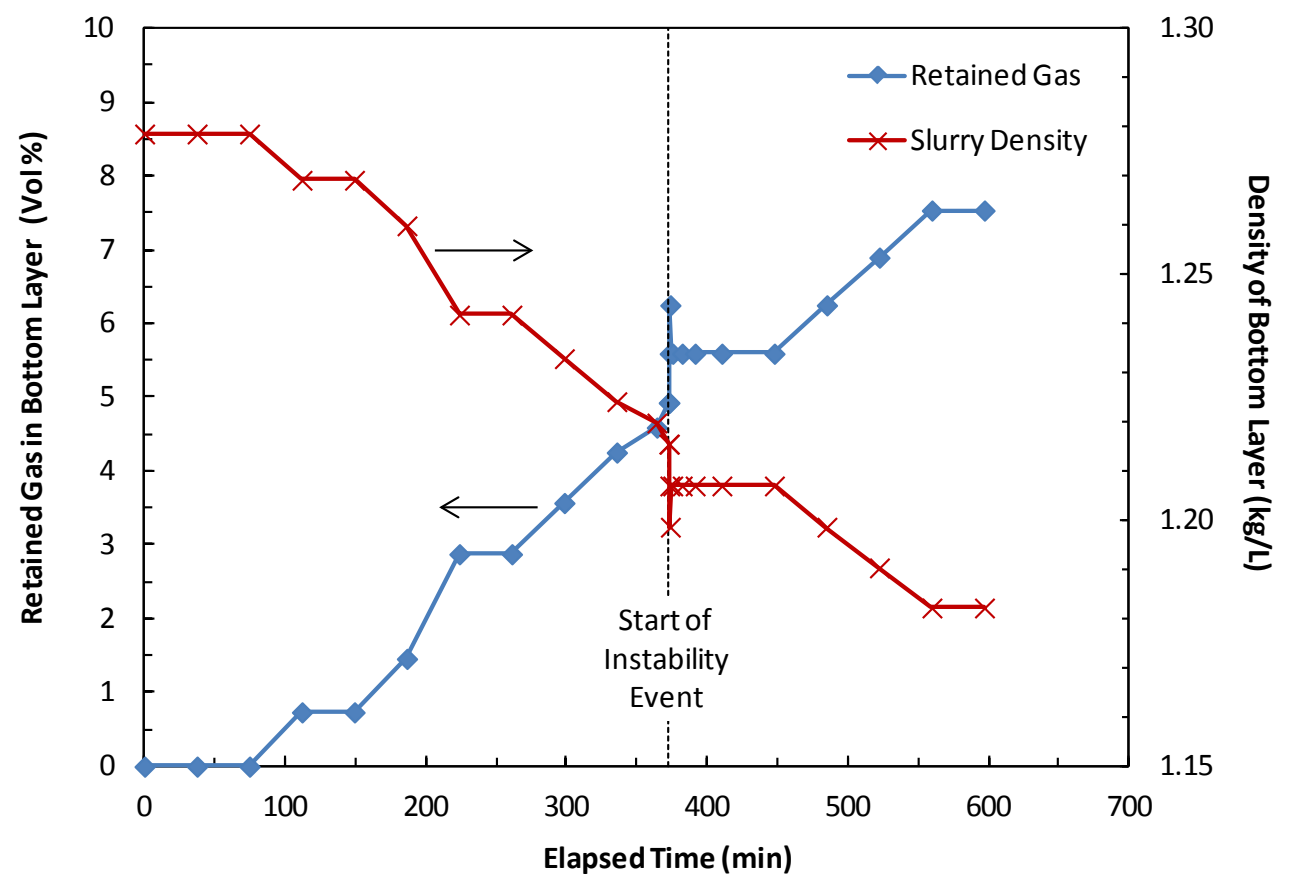

Figure 4.2. Retained Gas Volume Fraction and the Corresponding Calculated Density of the Bottom (Dyed) Slurry Layer during Scoping Test 1 (Elapsed Time is from the start of the test.)

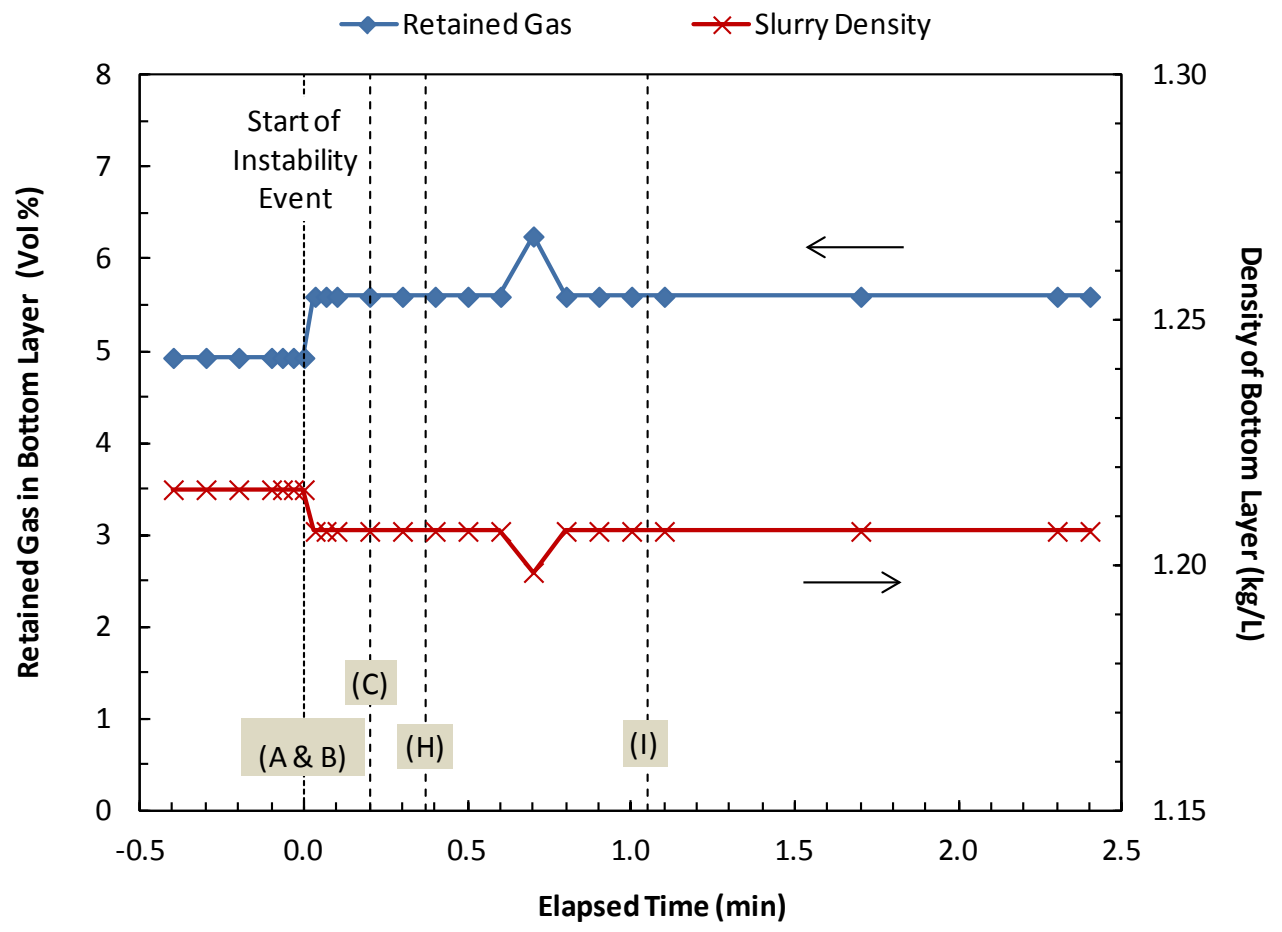

Figure 4.3. Detail of Instability Event in Scoping Test 1: Retained Gas Volume Fraction and the Corresponding Calculated Density of the Bottom (Dyed) Slurry Layer (Elapsed Time is from the start of the instability event; highlighted alphabetic labels refer to images in Figure 4.4.) 
In motion, the top-view video recording shows a low-amplitude wavelike (large wavelength relative to the vessel diameter) disturbance of the water layer from a few seconds before to the defined start of the instability event. This is shown by the difference in reflected light in images A (ET $-2 \mathrm{sec}$ ) and B (ET 0) of Figure 4.4. Detectable slurry movement initiated on the right side of the vessel ( 11 to 12 o'clock in top view) at the start of the event and can be seen more clearly in side-view images C and D (ET 12 and $13 \mathrm{sec}$ ). Motion of the upper slurry surface progressed to the center and left of the vessel as shown in the rise of slurry in side-view images E and F (ET 14 and $15 \mathrm{sec}$ ) while the right-side surface subsided. Side-view images F to H (ET 15 to $22 \mathrm{sec)}$ ) show that the supernatant water was significantly clouded by the ejection and settling of natural and dyed clay particulate. However, the top-view images B to H (ET 0 to $22 \mathrm{sec}$ ) show a very small gas release associated with the event as evidenced by the number of bubbles (small pock marks) and the development of an associated wet foam that covers a small fraction of the surface. Images at ET 63 and $72 \mathrm{sec}$ (I and J) depict the rise and fall of small gob of dyed slurry (a few centimeters across; far right of side view and 11 o'clock in top view). This suggests that the dyed clay migrated through or was exposed by movement of the natural clay at some time, and that the local gas fraction was sufficiently large to make the gob neutrally buoyant in water.

Both the level measurements and visual data show that there is no significant gas released during the event. In fact, the level data suggest that the volume fraction of retained gas increased slightly from just before to just after the start of the event, as shown in Figure 4.3. (The change is on the order of the resolution of level measurements.) An increase in gas volume is consistent with bubble rise and expansion. As discussed in Section 4.1, the maximum volume expansion due to reduction in hydrostatic pressure is expected to be $3.4 \%$ of the retained gas volume. For a retained gas fraction of $\sim 5 \mathrm{vol} \%$, the maximum volume increase would therefore be $\sim 0.2$ vol\%. Such expansion could in part explain the apparent increase in the volume of retained gas around the instability event.

Figure 4.3 shows that $\sim 5$ vol\% gas was retained at the start of the instability event. The corresponding calculated bulk density of the dyed bottom layer is $\sim 1.22 \mathrm{~kg} / \mathrm{L}\left(1220 \mathrm{~kg} / \mathrm{m}^{3}\right)$, well above neutral buoyancy in water, which would have required $\sim 22 \mathrm{vol} \%$ retained gas (Table 4.1 ). Therefore, the observed instability cannot be defined (in total) as a conventional buoyant displacement gas release event.

Rather, movement of the slurry layers in Scoping Test 1 is consistent with an inversion of the original bottom dyed slurry layer, in which gas bubbles are retained, and a denser natural-kaolin slurry layer above it. Substituting Equation (2.9) into Equation (2.8) and solving for gas void fraction $(\alpha)$ gives an estimated retained gas volume fraction of $\sim 10$ vol\% for a postulated RT instability for the initial conditions in Test 1 . While the retained gas fraction at the start of the instability was lower, an RT instability cannot be dismissed given the uncertainty in the instability model and other factors. For example, the initial irregularity in the interface of the two slurry layers in Test 1 could reduce the retained gas fraction needed to become unstable. Also, the shear strength of the bottom layer could decrease with increasing retained gas volume fraction, as was previously reported for bentonite clay/water slurries (Gauglitz et al. 1995). 

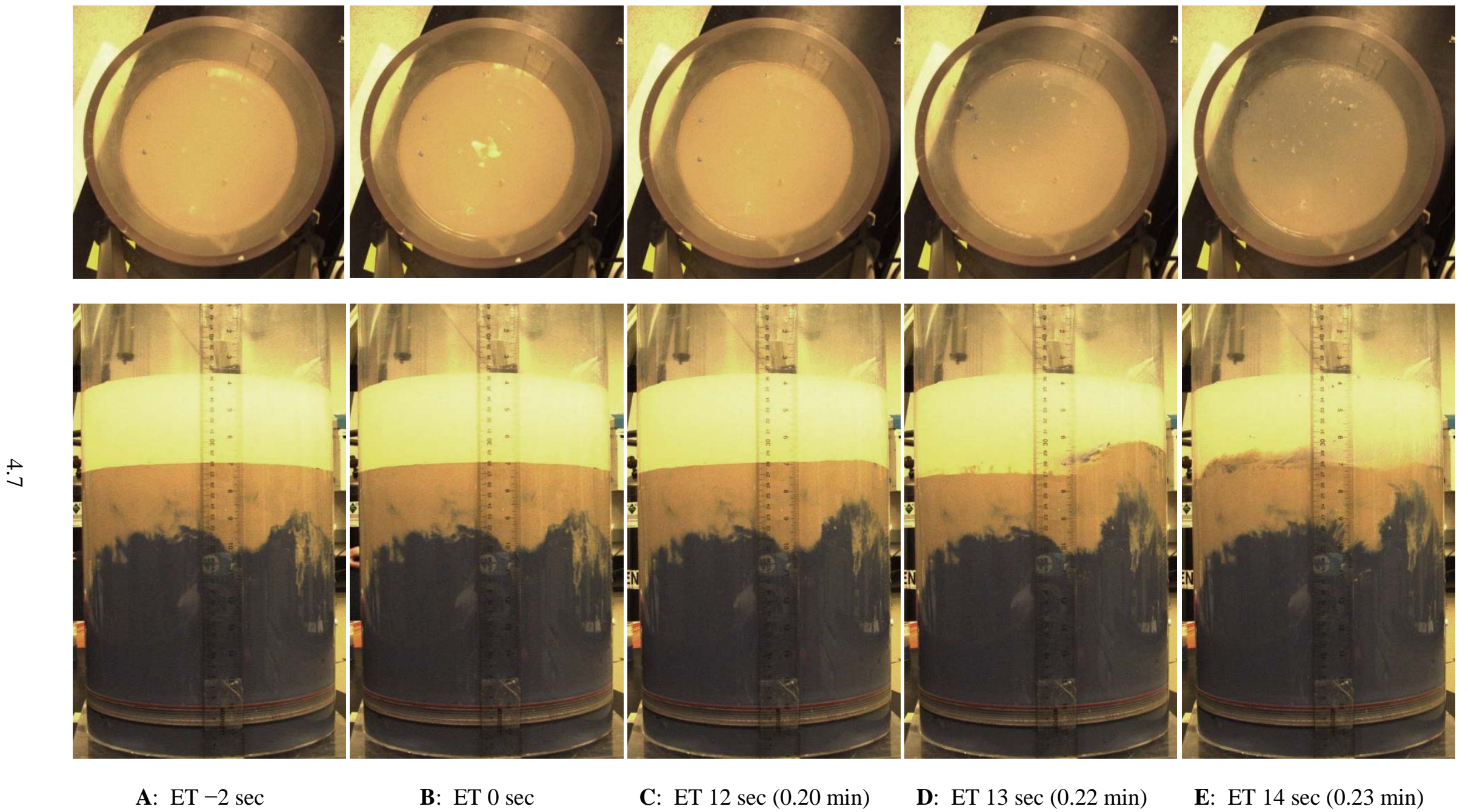

Figure 4.4. Progression of Top and Side Video Camera Images during and Following the Initial Instability Event in Test 1 (Elapsed Time, ET, is measured from the start of the instability event.) 

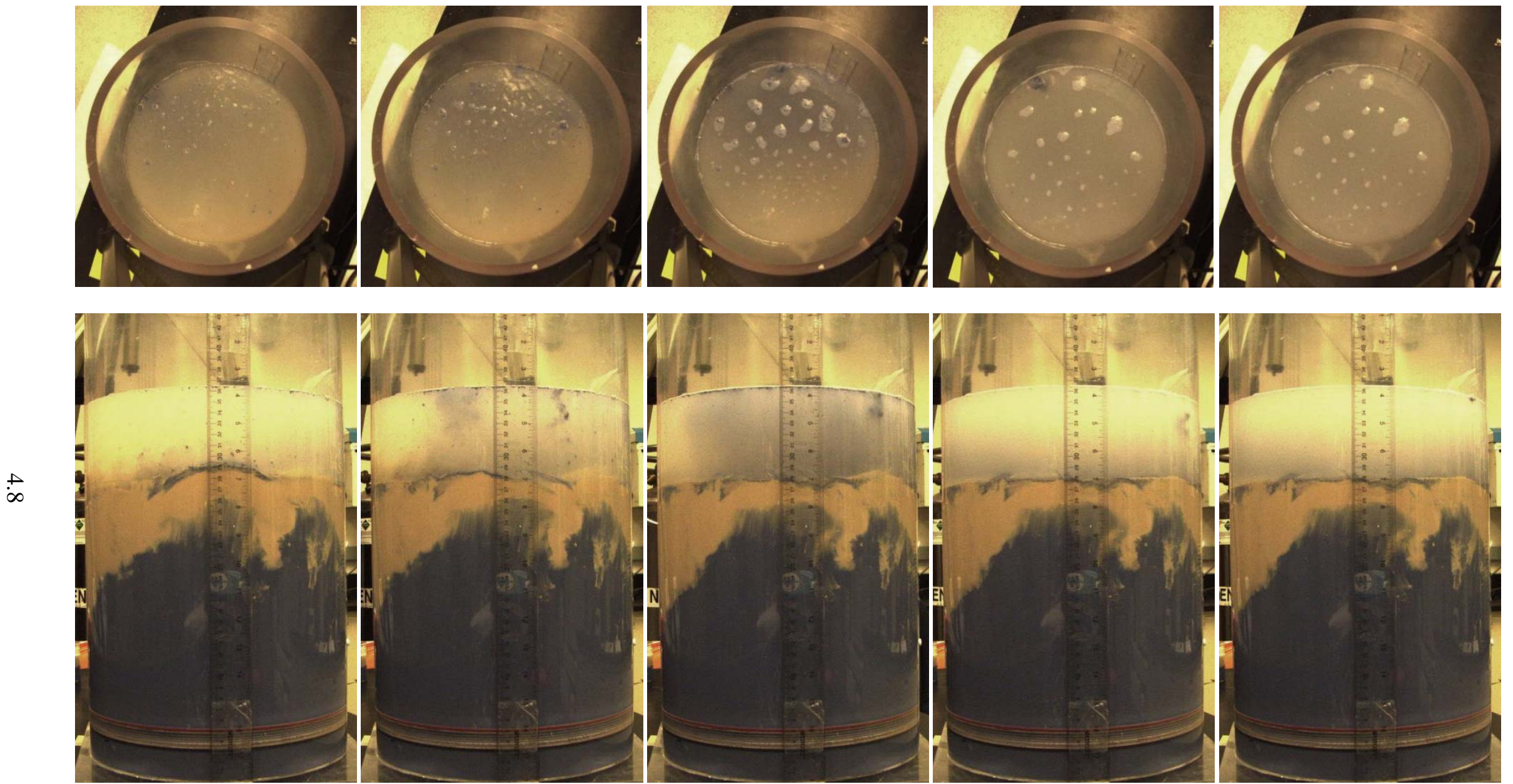

F: ET $15 \mathrm{sec}(0.25 \mathrm{~min})$

G: ET $16 \mathrm{sec}(0.27 \mathrm{~min})$

H: ET $22 \sec (0.37 \mathrm{~min})$

I: ET $63 \mathrm{sec}(1.05 \mathrm{~min})$

J: ET $72 \sec (1.20 \mathrm{~min})$

Figure 4.4. (contd) 


\subsubsection{Scoping Test 2: $\sim 32 \mathrm{~Pa}$ Shear Strength}

In Scoping Test 2, the kaolin slurry layers had initial shear strength of $\sim 32 \mathrm{~Pa}$ and density of $\sim 1.32 \mathrm{~kg} / \mathrm{L}$, as discussed in Section 4.1. For this test, the expected density of the kaolin layer at the onset of instability should exceed the water layer, and the expected motion should follow what is shown in the right-hand picture of Figure 3.4. In Figure 4.5, the left axis shows the volume fraction of retained gas in the dyed, bottom slurry layer during Test 2 and the right axis shows the slurry density. Unlike the first scoping test, there is no apparent lag preceding a steady increase in the volume fraction of retained gas. Movement of slurry signaling the onset of instability was detected visually at an elapsed time of $\sim 800$ min after the start of the test and at a retained gas fraction in the bottom slurry layer of $\sim 27$ vol\%., as shown in Figure 4.5. Figure 4.6 provides a more detailed view of the volume fraction of retained gas and slurry density in the period surrounding the initial instability event (elapsed time 0 in this figure). Following the start of the instability event, the water surface level increased slightly and then held steady for a couple of minutes before gas release was detected. As noted in the discussion of Scoping Test 1, the initial level increase could be due in part to bubble rise and a maximum expansion of $\sim 3.4 \%$ of the retained gas volume due to reduced hydrostatic pressure. For a retained gas fraction of $\sim 27 \mathrm{vol} \%$, the maximum increase due to expansion would be $\sim 0.9$ vol\%.

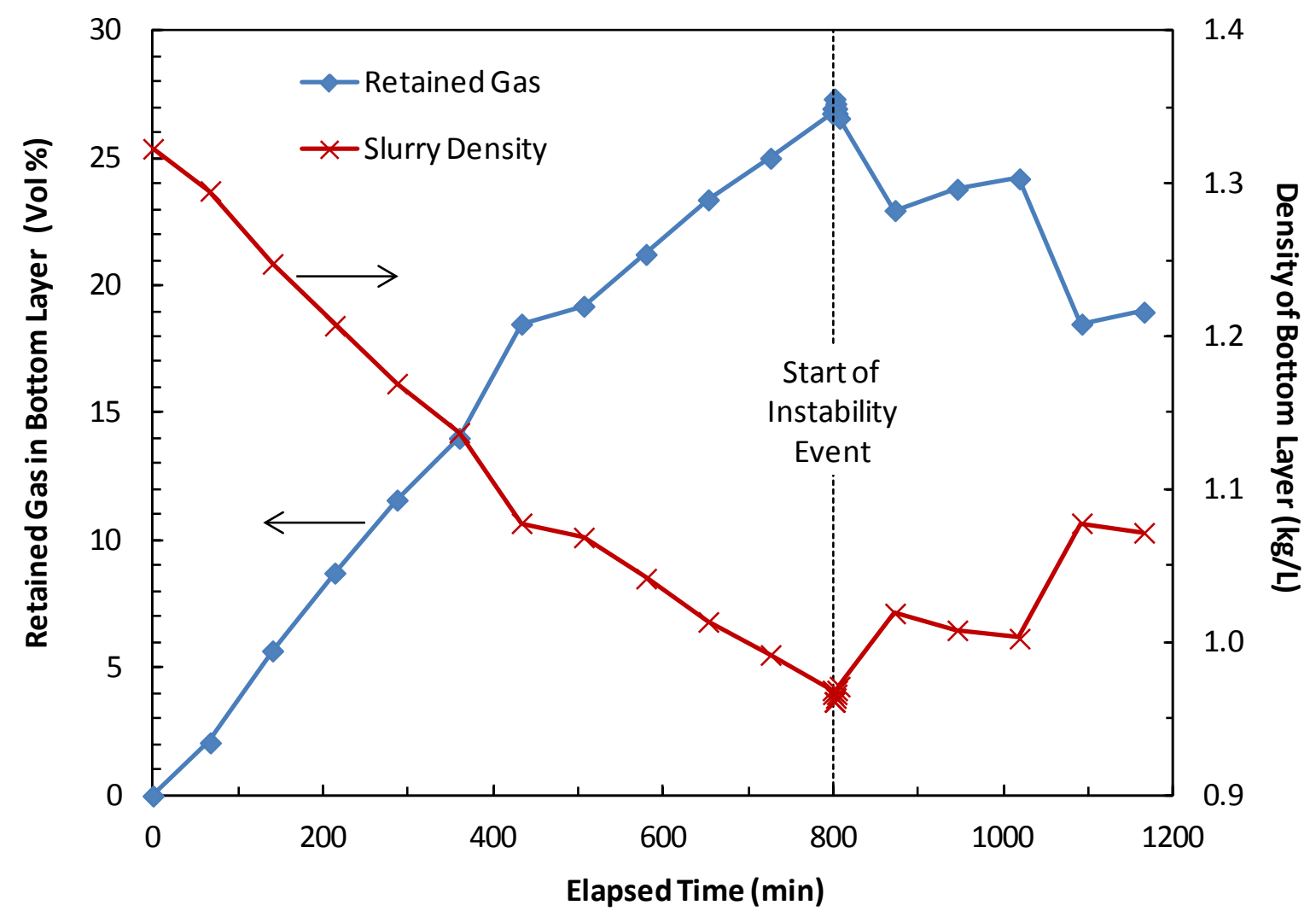

Figure 4.5. Retained Gas Volume Fraction and the Corresponding Calculated Density of the Bottom (Dyed) Slurry Layer during Scoping Test 1 (Elapsed Time is from the start of the test.) 
Figure 4.6 shows that $\leq 1$ vol\% of gas was released from the bottom slurry layer during and in the six minutes after the initial instability. However, Figure 4.5 indicates subsequent episodic releases of 3 to 5 vol\% interspersed with periods of gas retention and level growth. These additional events are not discussed further, as they are not pertinent to the assessment of a possible initiating RT instability.

Figure 4.7 shows a progression of top- and side-view video camera images from the start of the initial instability event and the $\sim 6$ min following. Like Scoping Test 1 , the alphabetic labels and corresponding time markers in the event detail plot, Figure 4.6, are keyed to images shown in Figure 4.7. Again, the ruler affixed to the side of the vessel is at one o'clock in the top-view images.

In Scoping Test 1, slurry motion along the vessel wall was the primary indicator of the onset of instability, whereas changes in the top view of the supernatant water surface and the slurry below it were more subtle. The primary indicator for the start of instability in Scoping Test 2 is reversed. Top-view images in Figure 4.7 show an apparently flat natural-kaolin surface (through hazy water) in image A (ET 0), a submerged, centrally located slurry dome in image B (ET $1 \mathrm{sec}$ ), and a mass of bubbly clay floating on the water surface with a perimeter of water between the floating mass and the vessel wall in image C (ET $2 \mathrm{sec}$ ). The primarily natural kaolin coloration of the slurry plume in the image $\mathrm{C}$ top view is thought to be due to entrainment of a thin natural clay "film" by the blue-dyed clay as it rose to the surface. This is supported, in part, by the image $\mathrm{C}$ side view in which dark colored gobs and swirling solids are observed. The cloud of non-floating dark solids in the water layer of side-view image C settled substantially 10 to 15 seconds later (image not shown), taking on an appearance similar to that in sideview image D (ET $43 \mathrm{sec}, 0.72 \mathrm{~min}$ ). Side-view image D also shows the development of large cracks (openings) in the natural-kaolin layer. These cracks started to become visible at an elapsed time of $\sim 25 \mathrm{sec}$ (0.42 min; image not shown) and continued to image E (ET $62 \mathrm{sec}, 1.03 \mathrm{~min}$ ) and beyond. Viewing the process in video motion shows that the formation of openings is due to sloughing of clay slurry off the walls, not expansion due to gas bubbles. Presumably a relatively thin layer of residual clay along the wall sloughed into a depression in the center of the slurry layer left by the mass of floating slurry. Completion of the sloughing process resulted in a condition similar to that shown in image $\mathrm{F}$ (ET $120 \mathrm{sec}, 2.0 \mathrm{~min}$ ). Also note that there is little change in the appearance of the floating layer in images D to F. Corresponding to images A to F of Figure 4.7, Figure 4.6 indicates that negligible gas volume was released in the first two minutes after the start of the instability. 


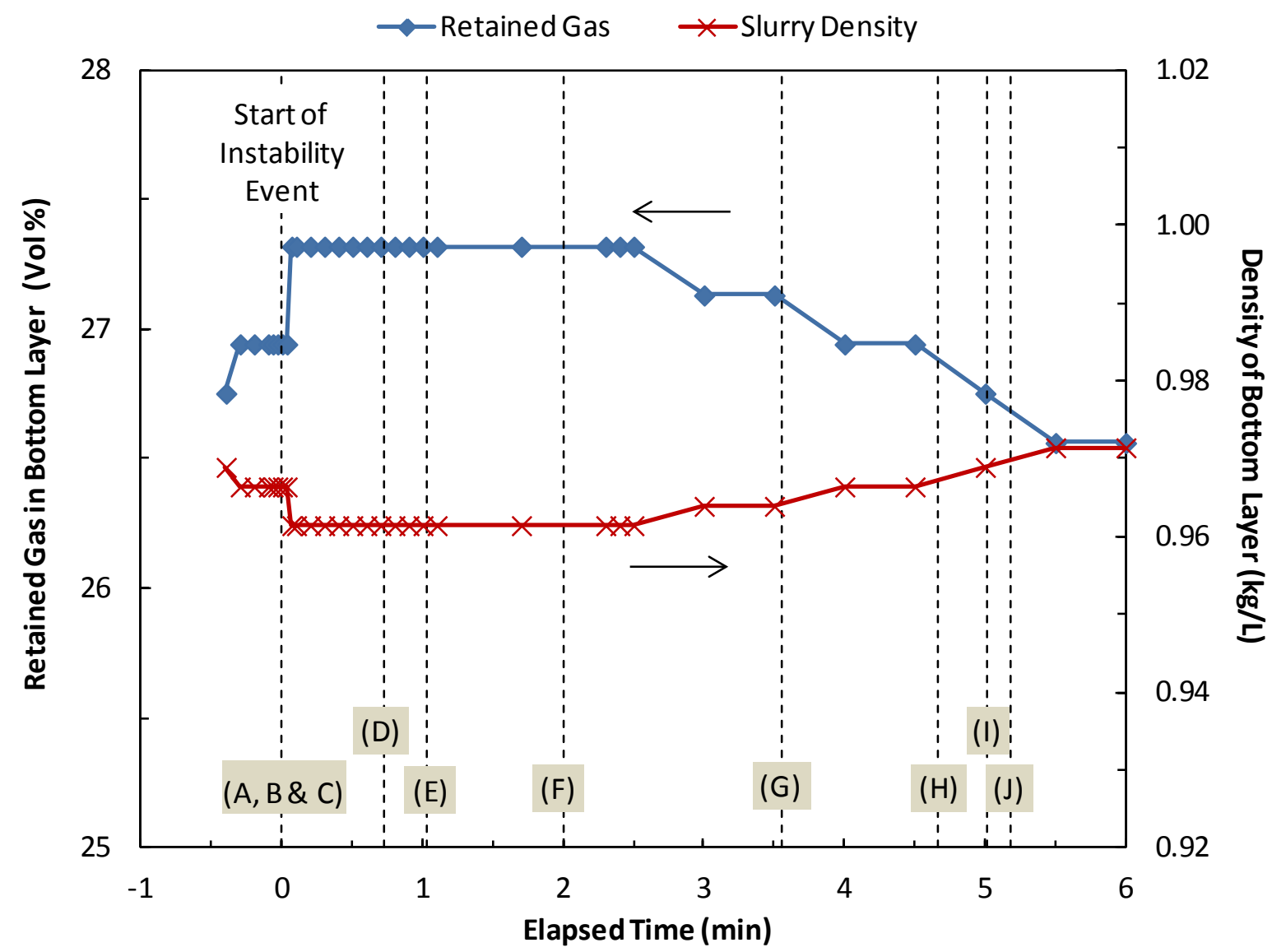

Figure 4.6. Instability Event Detail in Scoping Test 2: Retained Gas Volume Fraction and the Corresponding Calculated Density of the Bottom (Dyed) Slurry Layer (Elapsed Time from the start of the instability event; highlighted alphabetic labels refer to images in Figure 4.7) 


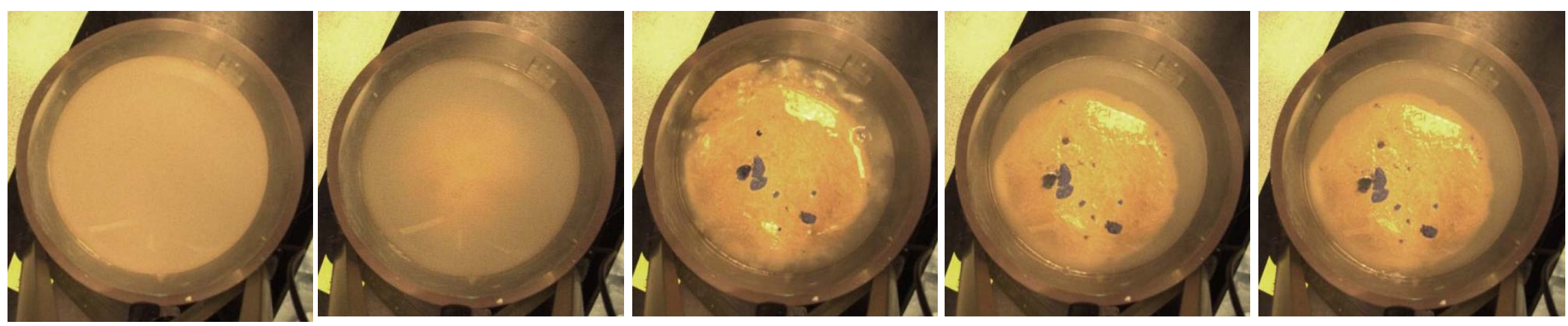

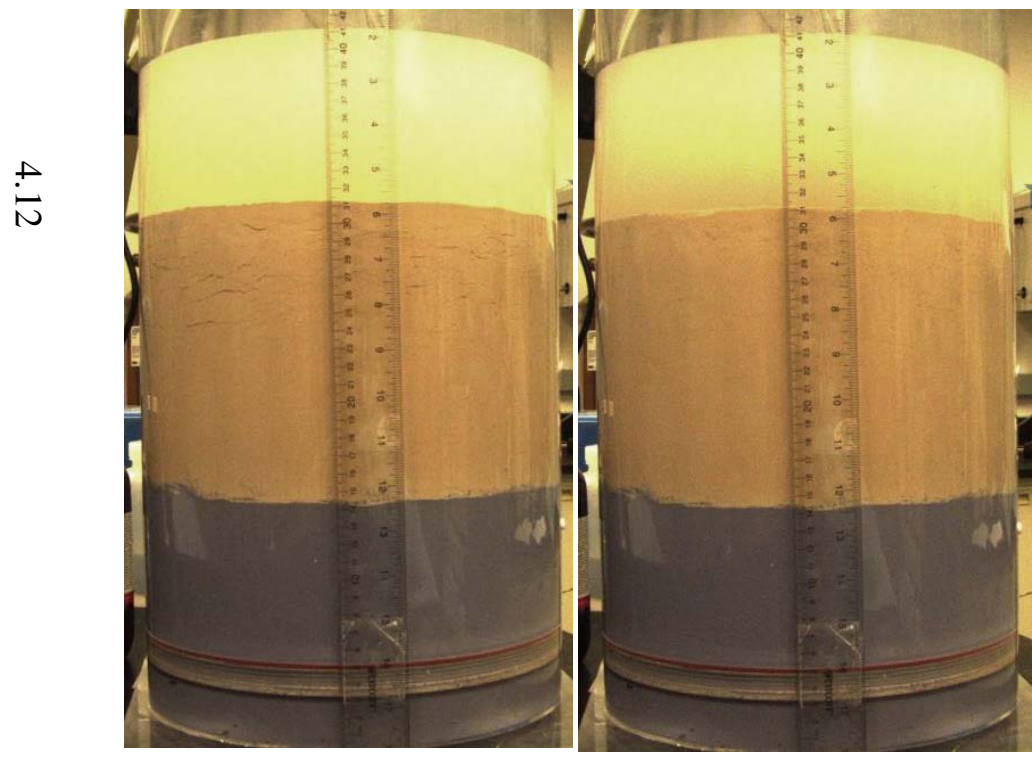

A: ET 0 sec
B: ET 1 sec

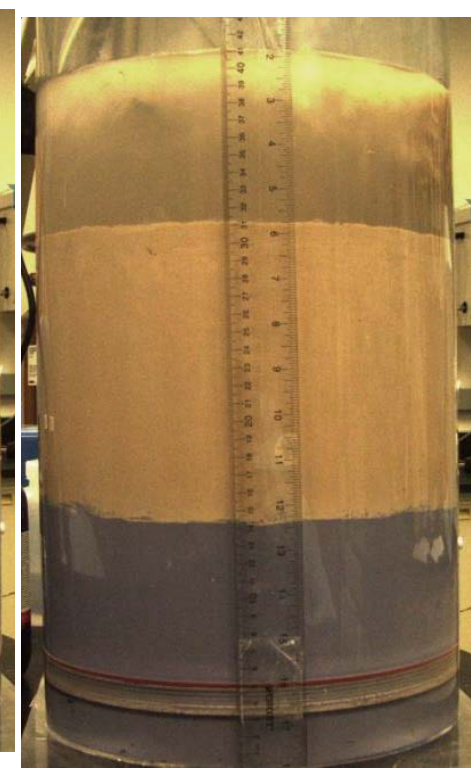

C: ET 2 sec

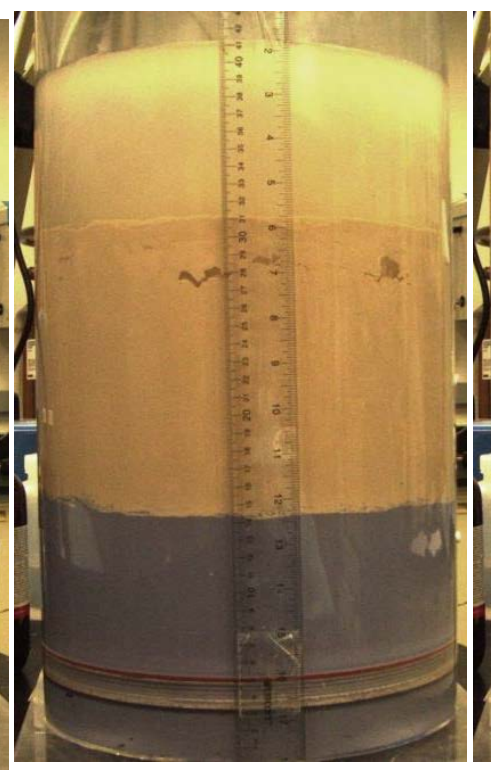

D: ET $43 \sec (0.72 \mathrm{~min})$

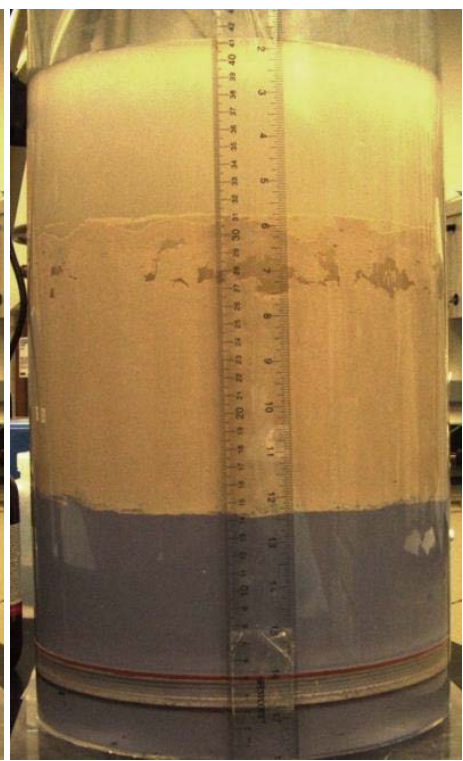

E: ET $62 \sec (1.03 \mathrm{~min})$

Figure 4.7. Progression of Top and Side Video Camera Images during and Following the Initial Instability Event in Test 2 (Elapsed Time, ET, from the start of the instability event.) 

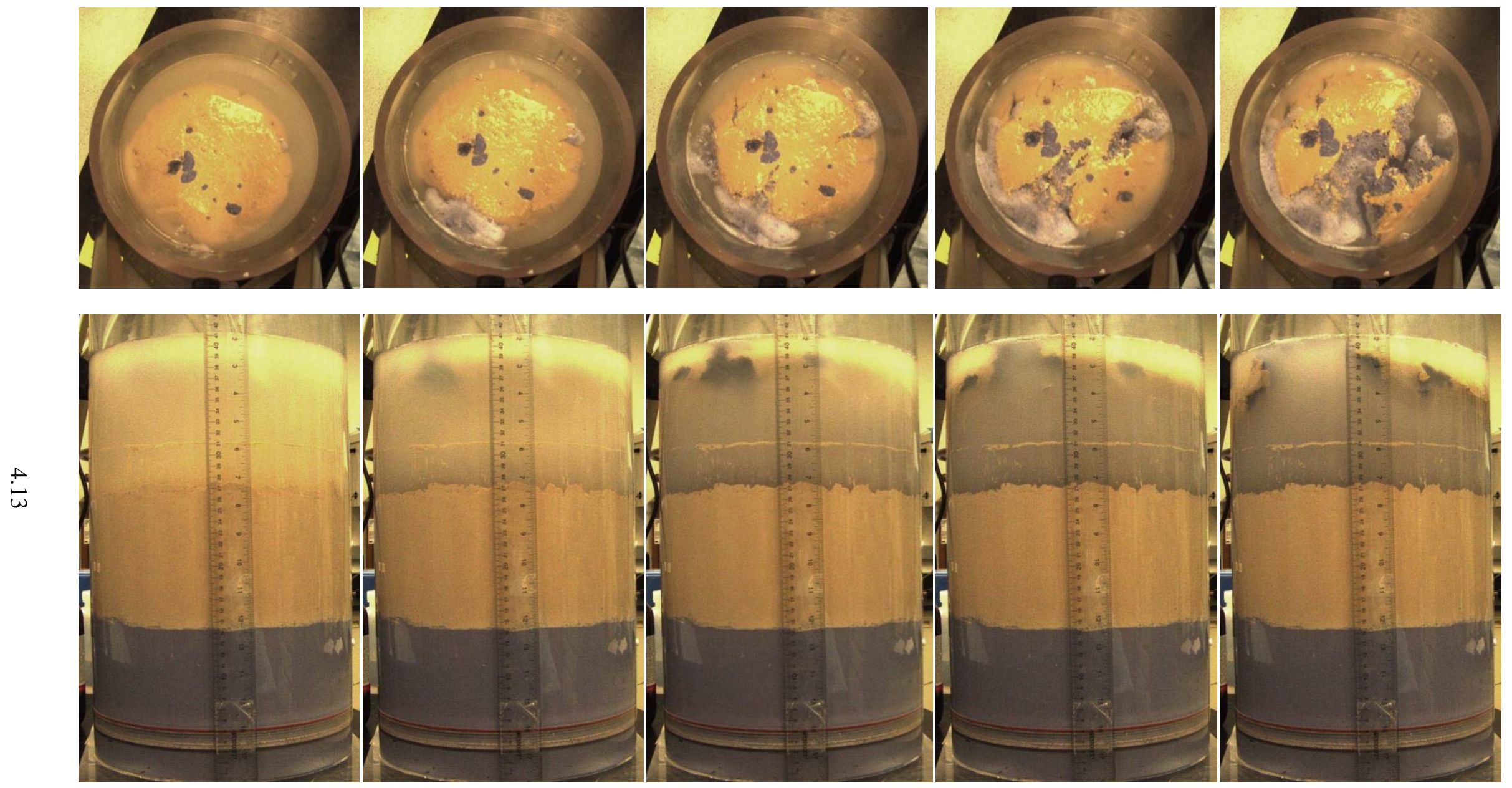

F: ET $120 \mathrm{sec}(2.00 \mathrm{~min})$

G: ET $213 \mathrm{sec}$ (3.55 min)

H: ET $280 \mathrm{sec}$ (4.67 $\mathrm{min})$

I: ET $301 \mathrm{sec}(5.02 \mathrm{~min})$

J: ET 311 sec (5.18 min) Figure 4.7. (contd) 
However, in the time frame spanned by images G (ET $213 \mathrm{sec}, 3.55 \mathrm{~min}$ ) to J (ET $311 \mathrm{sec}, 5.18 \mathrm{~min}$ ), gas release was detected in level measurements and noted visually. This sequence of images, $\mathrm{G}$ to J, shows: a) dark gobs rising and the formation and movement of a dark cloud in the water layer; b) gas bubbles releasing from the water surface (e.g., 7 o'clock in the top-view images); c) movement of the floating slurry in a direction from $\sim 7$ o'clock to $~ 1$ o'clock; and d) most significantly, fracturing of the floating plume between $\sim 7$ o'clock and $\sim 2$ o'clock and opening of a water-filled channel. Note that the freshly exposed surface along the fracture is primarily dyed slurry, supporting the hypothesis that the natural kaolin previously viewed on the surface was a thin entrained film.

Figure 4.5 and Figure 4.6 show that 27 vol\% gas was retained at the start of the instability event. The corresponding calculated bulk density of the dyed bottom layer is $\sim 0.97 \mathrm{~kg} / \mathrm{L}\left(970 \mathrm{~kg} / \mathrm{m}^{3}\right)$, thus allowing the bubbly slurry to become buoyant in water, as indicated by the floating mass in Figure 4.7. In contrast to Scoping Test 1, buoyant displacement cannot be readily dismissed as the possible basis of the initial instability in Scoping Test 2. However, possible initiation by RT instability is not automatically disqualified either. As was done for Scoping Test 1, substituting Equation (2.9) into Equation (2.8) and solving for gas void fraction gives an estimated retained gas volume fraction of $\sim 22$ vol\% for a postulated RT instability for the initial conditions in Test 2 . While the measured value at instability (27 vol\%) and the value estimated for neutral buoyancy in water ( 25 vol\%; Section 4.1) were higher, an RT instability cannot be dismissed given the uncertainty in the instability model and other factors. For example, consider that the change in the lower slurry layer levels did not match the change in water level. This is indicative of wall effects hindering the uniform rise of the slurry layers as gas was retained and suggests expansion of slurry more centrally in the vessel, resulting in doming. See, for example, images A to F of Figure 4.7 and the discussion thereof. These wall effects and the associated doming could functionally reduce the vessel diameter. To compensate for a reduced effective diameter, higher retained gas volume fractions would be needed to initiate an RT instability per Equation (2.8).

\subsection{Comparison of Test Results to Rayleigh-Taylor Instability Model}

In this section, the results of the two scoping tests in a 10 in $(0.254 \mathrm{~m})$ diameter vessel are compared to model predictions for the postulated onset of an RT instability for slurry layers of different density. As discussed above, instability in Scoping Test 1 ( 15 Pa shear strength and $\sim 1.28 \mathrm{~kg} / \mathrm{L}$ initial density) was detected at a retained gas volume fraction of $\sim 5$ vol\% in the bottom layer of kaolin slurry, whereas the predicted gas fraction necessary to reduce the density to initiate an RT instability is $\sim 10$ vol\%. Likewise, instability in Scoping Test 2 ( 32 Pa shear strength and $\sim 1.32 \mathrm{~kg} / \mathrm{L}$ initial density) was observed at a retained gas volume fraction of $\sim 27$ vol\% compared to a predicted gas fraction of $\sim 22 \mathrm{vol} \%$. The experimental results and the RT instability model predictions are shown in Figure 4.8. The figure also shows the retained gas volume fraction in slurry necessary to obtain neutral buoyancy in water for kaolin slurries of varying solid mass fraction, density, and shear strength. Figure 4.8 shows reasonable agreement between the experimental results and model predictions, considering the preliminary nature of the scoping tests and uncertainties in the model. For Test 1 , the early onset of the instability ( $\sim 5 \mathrm{vol} \%$ gas rather than the predicted onset at $10 \mathrm{vol} \%$ ) may be partly due to the irregularity in the interface between the two slurry layers when the test vessel was loaded and the experiment started.

For additional information on Figure 4.8, see the discussion of Figure 2.1 from which it was substantially derived. Unlike the basis figure, Figure 4.8 only includes curves for the $10 \mathrm{in}$. test vessel and a $75 \mathrm{ft}(22.9 \mathrm{~m})$ diameter Hanford waste storage tank. In the first scoping test, the target retained gas 
volume fraction (void difference) of $~ 10$ vol\% at instability was chosen to match a Hanford tank with waste having a shear strength of a few thousand Pa.

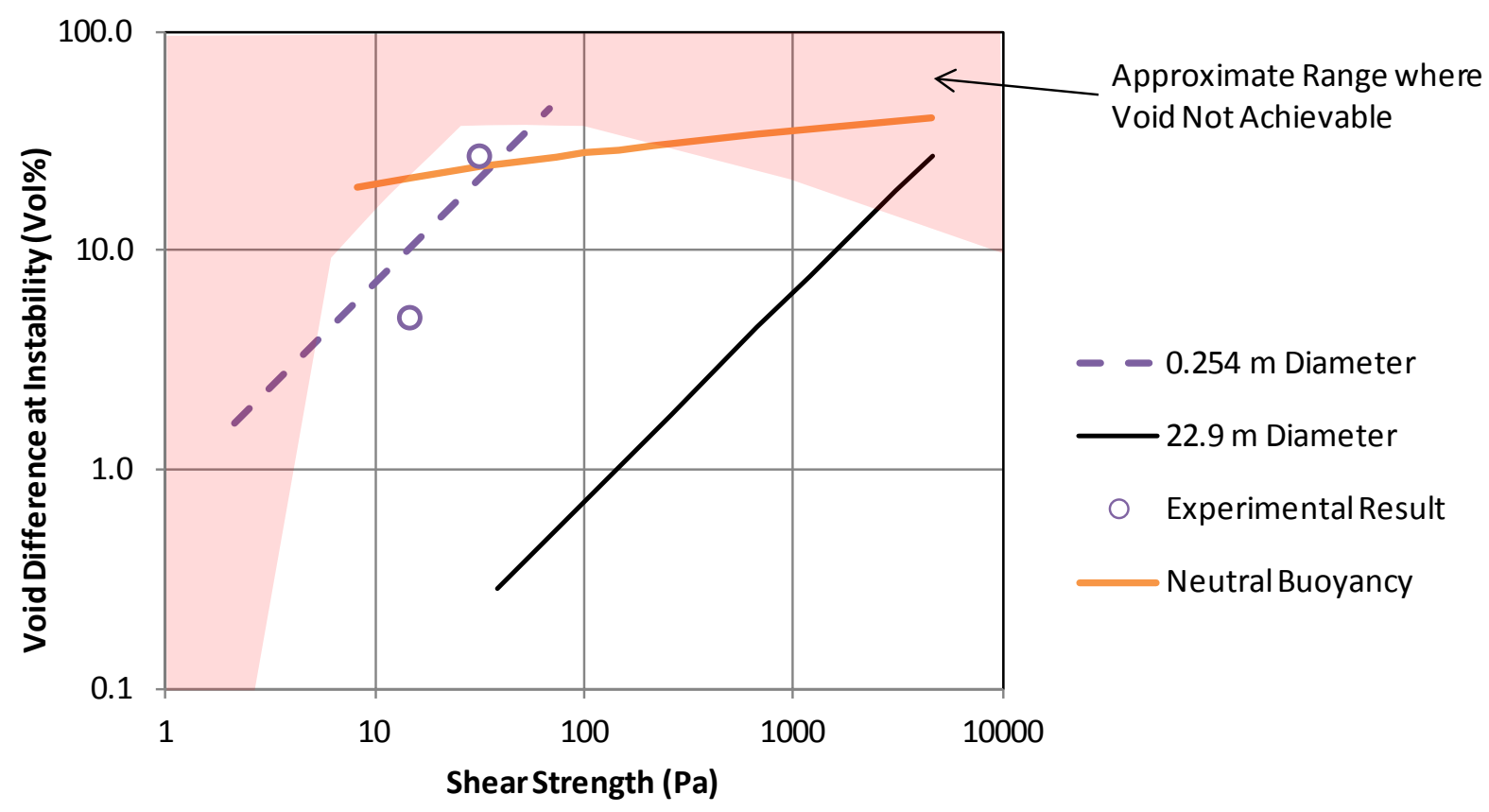

Figure 4.8. Comparison of Model-Predicted Retained Gas Fraction for an RT Instability to the Observed Onset of Instability in the Scoping Tests 



\subsection{Summary and Conclusions}

The purpose of this summary is to provide a review of the literature on RT instabilities with an emphasis on studies that are pertinent to RT instabilities between two solid materials of different bulk density that are formed from settled slurries. The potential for different gas retention in a sediment layer and gas release from the potential buoyant motion are also discussed and the results of preliminary experiments that demonstrate RT instabilities due to gas retention are summarized. The key conclusions are the following:

- The literature has useful theoretical and experimental results for estimating the void (or bulk density) difference between two layers that will induce the instability within tanks as well as the effects of yield stress and tank diameter. For soft solids such as waste slurries, previous experiments discussed in the literature have only been conducted with a slurry layer above a gas layer.

- The literature does not evaluate configurations with multiple layers of different bulk density. Configurations with multiple-layer systems are expected to be more stable than two layers with the same total difference in bulk density.

- In situ void fraction measurements in Hanford waste tanks, when combined with density measurements of de-gassed samples, identified examples in which the gas void fraction increases with depth, and the bulk density (including gas) decreases with depth, showing that that there is a potential for buoyant motion within waste sediment layers due to bulk density differences.

- Observations of bubble retention in experiments that had gas release pathways, which were generally filled with gas, did not show a noticeable difference in gas retention above and below the depth of an open gas channel. This suggests that a maximum channel depth does not indicate a depth below which there is an increase in gas retention. Additional experiments might confirm this observation.

- The magnitude of gas release from the potential buoyant motion within a sediment layer should decrease with increasing yield stress, and strong sediment layers with buoyant motion may have little gas release, but there are no data to support this anticipated behavior. Experiments and modeling studies could potentially quantify this expected behavior.

- Two scoping experiments were conducted with kaolin clay slurries. The first experiment used a kaolin slurry with a shear strength of about $15 \mathrm{~Pa}$ and the second experiment used a slurry with a shear strength of about $32 \mathrm{~Pa}$. Each experiment had two horizontal kaolin layers; hydrogen peroxide was added to the lower layer to generate gas bubbles, and thus reduce the bulk density, over the oneday duration of the experiment. Both experiments demonstrated buoyant motion once sufficient gas retention was obtained to initiate an instability. The gas void fraction required for the onset of the instability increased with increasing shear strength and the void fraction values at onset of instability roughly agree with the stability criterion for an RT instability.

- For both of the scoping experiments, the amount of gas release due to the buoyant motion was small. 



\subsection{References}

Barnes JF, PJ Blewitt, RG McQueen, KA Meyer, and D Venable. 1974. “Taylor Instability in Solids.” J. Appl. Physics 45:727-732.

Barnes JF, DH Janney, RK London, KA Meyer, and DH Sharp. 1980. “Further Experimentation on Taylor Instability in Solids.” J. Appl. Physics 91:4678-4679.

Chandrasekhar S. 1981. Hydrodynamic and Hydromagnetic Stability. Dover Publications, Inc. New York.

Crosato A. 1998. Non-Homogeneous Mud Structure at Consolidation: Drainage System Behavior. Technical Report DM16, Delft Hydraulics, the Netherlands.

Epstein M. 2002. “Taylor Instability of High-Strength Sludge Plug in a Storage Container.” Appendix in G Terrones and PA Gauglitz, Vessel-Spanning Bubble Formation in K-Basin Sludge Stored in Large Diameter Containers. PNNL-13805, Pacific Northwest National Laboratory, Richland, Washington.

Epstein M and PA Gauglitz. 2010. An Experimental Study of the Stability of Vessel-Spanning Bubbles in Cylindrical, Annular, Obround, and Conical Containers. FAI/09-272, Rev. 2, Fauske and Associates, LLC, Burr Ridge, Illinois.

Gauglitz PA, SD Rassat, MR Powell, RR Shah and L.A. Mahoney. 1995. Gas Bubble Retention and its Effect on Waste Properties: Retention Mechanisms, Viscosity, and Tensile and Shear Strength. PNL-10740, Pacific Northwest Laboratory, Richland, Washington.

Gauglitz PA, WC Buchmiller, JJ Jenks, J Chun, RL Russell, AJ Schmidt, and MM Mastor, Jr. 2010. The Disruption of Vessel-Spanning Bubbles with Sloped Fins in Flat-Bottom and 2:1 Elliptical-Bottom Vessels. PNNL-19345 Rev 0, Pacific Northwest National Laboratory, Richland, Washington.

Gauglitz PA, WC Buchmiller, SG Probert, AT Owen and Brockman. 2012. Strong-Sludge Gas Retention and Release Mechanisms in Clay Simulants. PNNL-21167, Rev 0, Pacific Northwest National Laboratory, Richland, Washington.

Hedengren DC, KM Hodgson, WB Barton, CW Stewart, JM Cuta, and BE Wells. 2000. Data Observations on Double-Shell Flammable Gas Watch List Tank Behavior. RPP-6655. CH2M HILL Hanford Group Inc., Richland, Washington.

Hedengren DC, TA Hu, MA Kufahl, DJ McCain, CW Stewart, JL Huckaby, LA Mahoney and KG Rappe. 2001. Data and Observations of Single-Shell Flammable Gas Watch List Tank Behavior. RPP-7249, CH2M HILL Hanford Group Inc., Richland, Washington.

Kull HJ. 1991. “Theory of the Rayleigh-Taylor Instability.” Phys. Rep. 206:197-325.

Lewis DJ. 1950. "The Instability of Liquid Surfaces when Accelerated in a Direction Perpendicular to Their Planes. II.” Proc. Royal Soc. A 202:81-96. 
Meacham JE. 2010. Gas Retention and Release from Hanford High Shear Strength Waste. RPP-RPT-26836, Rev. 0, Washington River Protection Services LLC, Richland, Washington.

Meacham JE and NW Kirch. 2013. Initial Assessment for Potential Gas Release Events in Hanford Site Deep Sludge Double-Shell Tank Waste. RPP-RPT-54305, Rev. 0, Washington River Protection Solutions, Richland, Washington.

Meyer PA and CW Stewart. 2001. Preventing Buoyant Displacement Gas Release Events in Hanford Double-Shell Waste Tanks. PNNL-13337, Pacific Northwest National Laboratory, Richland, Washington.

Meyer PA, ME Brewster, SA Bryan, G Chen, LR Pederson, CW Stewart, and G Terrones. 1997. Gas Retention and Release Behavior in Hanford Double-Shell Waste Tanks. PNNL-11536, Rev. 1, Pacific Northwest National Laboratory, Richland, Washington.

Miles JW. 1966. “Taylor Instability of a Flat Plate.” General Dynamics, General Atomic Division Report GAMD-7335 (August).

Piriz AR, JJ Lopez Cela, and OD Cortazar. 2005. "Rayleigh-Taylor Instability in Elastic Solids.” Phys. Rev. E 72:056313-1-056313-9.

Piriz AR, JJ Lopez Cela, and NA Tahir. 2009. "Rayleigh-Taylor Instability in Elastic-Plastic Solids.” J. Appl. Phys. 105:116101-1-116101-3.

Rayleigh, Lord (JW Strutt). 1883. "Investigation of the Character of the Equilibrium of an Incompressible Heavy Fluid of Variable Density.” Proc. London Math. Soc. 14:170-177.

Rassat SD, SM Caley, PR Bredt, PA Gauglitz, DE Rinehart, and SV Forbes. 1998. Mechanisms of Gas Bubble Retention and Release: Experimental Results for Hanford Single Shell Waste Tanks 241-A-101, 241-S-106, and 241-U-103. PNNL-11981, Pacific Northwest National Laboratory, Richland, Washington.

Sharp DH. 1984. “An overview of Rayleigh-Taylor Instability.” Physica D 12:3-10.

Stewart CW, SA Hartley, PA Meyer and BE Wells. 2005. Predicting Peak Hydrogen Concentrations from Spontaneous Gas Releases in Hanford Waste Tanks. PNNL-15238, Pacific Northwest National Laboratory, Richland, Washington.

Stewart CW, JM Alzheimer, ME Brewster, G Chen, RE Mendoza, HC Reid, CL Shepard, and G Terrones. 1996a. In Situ Rheology and Gas Volume in Hanford Double-Shell Waste Tanks. PNL-11296, Pacific Northwest National Laboratory, Richland, Washington.

Stewart CW, ME Brewster, PA Gauglitz, LA Mahoney, PA Meyer, KP Recknagle, and HC Reed. $1996 \mathrm{~b}$. Gas Retention and Release Behavior in Hanford Single-Shell Tanks. PNNL-11391, Pacific Northwest National Laboratory, Richland, Washington.

Taylor GI. 1950. “The Instability of Liquid Surfaces when Accelerated in a Direction Perpendicular to Their Planes. I.” Proc. Royal Soc. A 201:192-196. 
Terrones G. 2005. "Fastest Growing Linear Rayleigh-Taylor Modes at Solid/Fluid and Solid/Solid Interfaces.” Phys. Rev. E 71:036306-1-036306-11.

Terrones G. 2007. "Elastic Stability and the Onset of Plastic Flow in Accelerated Solid Plates.” J. of Appl. Phys. 102, 034908.

Van Kessel T. 1999. Evaluation Report of Depot Modelling. Technical Report Z2517/DM24, Delft Hydraulics, the Netherlands.

Van Kessel T and WGM van Kesteren. 2002. "Gas Production and Transport in Artificial Sludge Depots.” Waste Management 22(1):19-28.

Weber RA. 2009. Methodology and Calculations for the Assignment of Waste Groups for the Large Underground Storage Tanks at the Hanford Site. RPP-10006, Rev. 8, Washington River Protection Solutions, Richland, Washington.

Wells BE, RL Russell, LA Mahoney, GN Brown, DE Rinehart, WC Buchmiller, EC Golovich, and JV Crum. 2010a. Hanford Sludge Simulant Selection for Soil Mechanics Property Measurement. PNNL-19250, Pacific Northwest National Laboratory, Richland, Washington.

Wells BE, JJ Jenks, G Boeringa, NN Bauman, and AD Guzman. 2010b. Lateral Earth Pressure at Rest and Shear Modulus Measurements on Hanford Sludge Simulants. PNNL-19829, Pacific Northwest National Laboratory, Richland, Washington.

Winterwerp JC and WGM van Kesteren. 2004. "Introduction to the Physics of Cohesive Sediment in the Marine Environment.” Developments in Sedimentology, Vol. 56, Elsevier, New York. 



\section{Distribution}

No. of

Copies

\section{OFFSITE}

$1 \quad$ M Epstein

Fauske and Associates, LLC

1118 3rd Street, \#308

Santa Monica, CA 90403

1 G Terrones

Applied Physics Division (X-4)

MS: T086

Los Alamos National Laboratory

Los Alamos, NM 87545

3 Savannah River National Laboratory

Savannah River Site

Aiken, SC 29808

D Koopman

B Willmarth

C Nash

1 RD Holtz

Civil \& Environmental Engineering

University of Washington

201 More Hall, Box 352700

Seattle, WA 98195-2700
No. of

$\underline{\text { Copies }}$

\section{ONSITE}

7 Washington River Protection Solutions

WB Barton $\quad$ S7-90

R Calmus H8-04

JM Grigsby $\quad$ S7-90

NW Kirch R2-58

DB Little R2-58

JE Meacham R2-58

TL Sams R2-52

15 Pacific Northwest National Laboratory

WC Buchmiller K6-26

PA Gauglitz (10) K7-15

SD Rassat K6-28

DR Rector K7-15

BE Wells K7-15

Project Records 




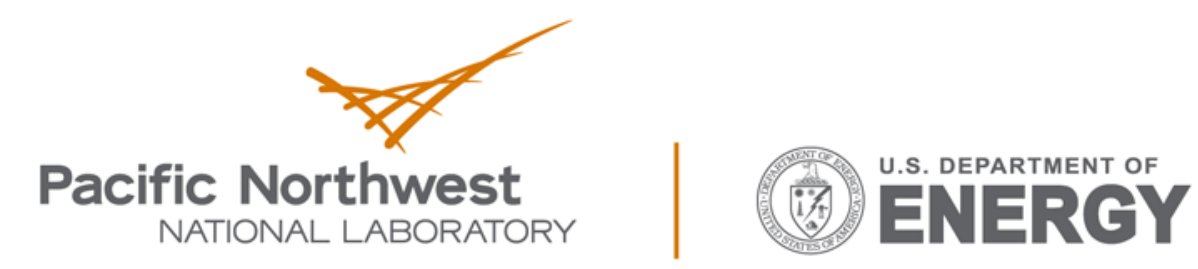

Proudly Operated by Battelle Since 1965

902 Battelle Boulevard

P.O. Box 999

Richland, WA 99352

1-888-375-PNNL (7665)

www.pnnl.gov 